
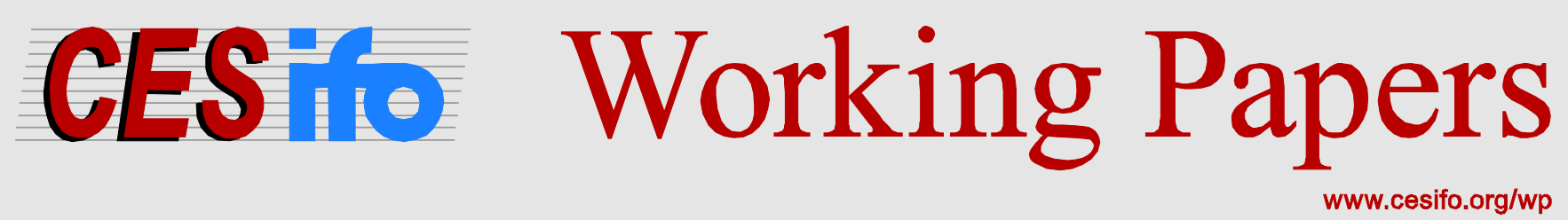

\title{
Fair Intergenerational Utilitarianism: Risk, its Resolution over Time, and Discounting
}

\author{
Paolo G. Piacquadio
}

CESIFO WORKING PAPER NO. 5143

CATEgory 2: Public CHOICE

DECEMBER 2014
An electronic version of the paper may be downloaded
- from the SSRN website: Www.SSRN.com
- from the RePEc website: Www.RePEc.org
- from the CESifo website: www.CESifo-group.org/wp




\title{
Fair Intergenerational Utilitarianism: Risk, its Resolution over Time, and Discounting
}

\begin{abstract}
The paper reexamines the welfare economics of intergenerational risk. Risk and its resolution over time are modeled as a decision tree: in each period, the consumption of the current oneperiod living generation is to be traded-off against uncertain benefits of future generations; as time passes, the planner observes the realized shocks and becomes more informed about the economy. The characterized class of criteria, named fair intergenerational utilitarian, measures social welfare in terms of the ratio between the allocated consumptions and an endogenously-determined equitable reference. This allows social preferences to (i) disentangle aversion to intergenerational inequality from aversion to risk, (ii) exhibit a preference for early resolution of risk, (iii) show different discounting formulas depending on the magnitude of risk and on the timing of its resolution, and (iv) avoid extreme policy recommendations in the presence of fat-tailed catastrophic events.
\end{abstract}

JEL-Code: D630, D810, H430, Q540, Q560.

Keywords: intergenerational justice, timing of risk resolution, social ordering, discounting.

Paolo G. Piacquadio
ESOP and Department of Economics
University of Oslo
Moltke Moes vei 31
Norway - 0317 Olso
p.g.piacquadio@econ.uio.no

The author thanks Geir Asheim, Claude d'Aspremont, Johannes Emmerling, Marc Fleurbaey, Reyer Gerlach, Peter Hammond, Bård Harstad, Francois Maniquet, Frikk Nesje, Christian Traeger and the audiences at UiO (Oslo), BEEER (Bergen), FEEM (Milan, Venice), SSCW (Boston), SDU (Odense), CESifo (Munich), NMBU (Ås), and CORE (Louvain-la-Neuve). This paper is part of the research activities at the Centre for the Study of Equality, Social Organization, and Performance (ESOP) at the Department of Economics at the University of Oslo. ESOP is supported by the Research Council of Norway through its Centres of Excellence funding scheme, project number 179552. The author also acknowledges a travel grant by the Norwegian Central Bank. 


\section{INTRODUCTION}

This paper addresses the assessment of risky intergenerational social situations. Let an event tree describe time, uncertainty, and the revelation of information. At each node, available capital is used as input in production; the produced output is then shared between consumption of the current one-period living generation (named conditional generation) and capital-savings for later use. The later evolution of technology is unknown at the moment this decision is taken and forces the society to trade-off the certain consumption of the current generation with uncertain consumptions of future ones.

The main result is to axiomatically characterize a new family of social orderings for intergenerational models of risk: the fair intergenerational utilitarian criteria rank alternatives (or prospects) based on the information about technological risk and the timing of its resolution. The normative reasoning leading to this family comprises three steps, hereafter briefly described.

Step 1 associates an equitable reference to each risky intergenerational situation. This reference, named fair prospect, is selected by means of an allocation rule and interprets the ideal of distributional fairness: it is the most appealing prospect in terms of equity and efficiency. I shall postpone the discussion on how to construct the equitable reference: the welfare representation of the fair intergenerational utilitarian criterion is independent of the fairness principles the fair prospect embodies. ${ }^{1}$

Step 2 formalizes the ethical concerns for intergenerational inequality and risk. The first axiom, intergenerational equity, is inspired by the Pigou-Dalton transfer principle. $^{2}$ Assume a generation is, at each possible history, better-off than at the fair prospect while another generation is, at each possible history, worse-off than at the fair prospect. Then, a (discounted) transfer, uniform across histories, that redistributes consumptions from the first to the second generation is welfare improving. Such transfer reduces -without reversing - the gap between the initial allocation of resources and the fair prospect, while leaving all other generations unaffected.

The second axiom, risk-balancing, is a mean-preserving redistributive transfer, related to Rothschild and Stiglitz (1970). Assume a generation is treated as follows:

\footnotetext{
${ }^{1}$ An attractive reference is an efficient prospect that assigns to each conditional generation the same consumption; however, since technology differs across possible histories, this reference generally does not exist and a tension between equity and efficiency arises. I discuss how to resolve this tension in Section 5 .

${ }^{2}$ Following an intuition of Pigou (1912), Dalton (1920) suggested that a transfer of wealth from a richer to a poorer person, to the limit that this transfer does not reverse the inequality, brings to a more equitable distribution of resources.
} 
at one possible history the assigned consumption is larger than at the fair prospect, while at another possible history the consumption is smaller. Then a mean preserving transfer from the former to the latter is welfare improving. As before, such transfer reduces -without reversing - the gap between the initial allocation of resources and the fair prospect.

Step 3 completes the characterization result by introducing the following requirements. Strict monotonicity entails sensitivity to the consumption of each conditional generation: assigning more consumption leads to a socially improved prospect. Continuity and two separability conditions, one over generations and one over possible histories, provide functional representability and additive separability of the welfare measure. Independence to proportional changes of the fair prospect ensures that what matters for the identification of the richer/poorer relations are their relative, and not absolute, consumptions.

The above described axioms uniquely characterize the fair intergenerational utilitarian criterion, which I shall describe next. Let $D$ denote an intergenerational resource distribution problem: it specifies the event tree $N$, the technology at each node, the cumulative probability $\pi_{n}>0$ that each node $n \in N$ is reached from the initial node, and the initial capital stock. The fair intergenerational utilitarian criterion measures the well-being of each conditional generation $n$ in terms of its assigned consumption, $c_{n}$, relative to the fair prospect, $x_{n}$. The welfare of generation $t$ is a weighted CES aggregation across conditional generations $n \in N_{t}$ of such relative consumptions:

$$
v_{t}(c ; D) \equiv\left(\sum_{n \in N_{t}} \frac{\pi_{n} x_{n}}{\sum_{\bar{n} \in N_{t}} \pi_{\bar{n}} x_{\bar{n}}}\left(\frac{c_{n}}{x_{n}}\right)^{\gamma}\right)^{\frac{1}{\gamma}} \quad \text { with } \gamma<1 .
$$

Intergenerational social welfare is given by the risk-adjusted discounted sum of a CRRA transform of each generation's welfare. The risk-adjusted discount factor at $t$ is:

$$
\tilde{\beta}_{t} \equiv \beta^{t}\left(\frac{\sum_{n \in N_{t}} \pi_{n} x_{n}}{x_{0}}\right) .
$$

It consists of two components: the standard exponential time discount factor $\beta^{t}$; and a risk-correction factor, measured by the average consumption that would be assigned 
to generation $t$ at the fair prospect $x$ with respect to what would be assigned to generation 0 (facing no risk). ${ }^{3}$ Formally, the social welfare of prospect $c$ for distribution problem $D$ is ordinally equivalent to:

$$
V(c ; D) \equiv \sum_{t \in T} \tilde{\beta}_{t} \frac{\left(v_{t}(c ; D)\right)^{1-\rho}}{1-\rho} \quad \text { with } \rho \geq 0 .
$$

The fair prospect $x$ plays an important role in the measurement of intergenerational social welfare: inequalities across time and histories are measured by how much the distribution of consumptions differs from it. ${ }^{4}$ When the benefit of redistributing resources over time (allowing for more consumption to be allocated) is larger than the cost of increasing intergenerational inequality (measured by the parameter $\rho$ ), some inequalities across generations are recommendable. Similarly, if some inequality across histories allows distributing more consumption to the conditional generations, this inequality might be socially acceptable, depending on the society's aversion to risk (measured by the parameter $\gamma$ ). At the limit for infinite aversion to intergenerational inequality $(\rho \rightarrow \infty)$ and infinite aversion to risk $(\gamma \rightarrow-\infty)$, the welfare cost of deviating from the reference explodes: the society would measure welfare by the relative consumption $\frac{c_{n}}{x_{n}}$ of the worst-off conditional generation, i.e. $V(c ; D)=\min _{n \in N} \frac{c_{n}}{x_{n}}$.

I suggest the fair prospect to be selected based on two appealing properties: maximality requires the prospect to be non-wasteful; interim egalitarianism demands the consumption assigned to each conditional generation to be socially as desirable as the risky consumption assigned to the later conditional generations at the nodes that can still realize. This condition is related to and somewhat combines the ex-ante egalitarian concern for fairness (Diamond (1967)) - giving agents equal changes is socially desirable- and the ex-post egalitarian concern for distributional equity (Broome (1984); Fleurbaey (2010)) -reducing inequalities among agents at each possible state of nature is socially desirable to. These have emerged in the debate around expected utilitarianism, first characterized by Harsanyi (1953; 1955). Despite the differences

\footnotetext{
${ }^{3}$ In the online appendix, this discount factor is endogenously obtained: it is singled out by the fairness axioms in a more general model, where the possibility of human race extinction is accounted for.

${ }^{4}$ The idea is somewhat related to the modeling of consumer's behavior in prospect theory, see Kahneman and Tversky (1979), Sugden (2003), and Kòszegi and Rabin (2006). Apart from the normative social perspective, the independence to proportional changes of the fair prospect implies that inequalities are relative in nature and thus avoids kinks and convex/concave welfare representations, typical of prospect theory.
} 
with Harsanyi's setting -a static framework in which social welfare aggregates individual preferences over assigned lotteries- similar egalitarian arguments can be raised for the intergenerational model and ethically justify interim egalitarianism.

When this specific reference is chosen for the fair intergenerational utilitarian criterion, the evaluation of risky intergenerational prospects delivers interesting consequences.

First, it not only disentangles risk aversion from intergenerational inequality aversion, ${ }^{5}$ but also distinguishes between two different sources of risk: intrinsic risk is unavoidable even at the egalitarian reference and is determined, together with the planner's aversion to it, by the specific resource distribution problem; option risk, instead, arises only if the planner decides to deviate from the fair prospect (the sensitivity to such deviations is measured by the elasticity parameter $\gamma$ in the fair intergenerational utilitarian criterion).

Second, the planner values early resolution of uncertainty for allowing a more equitable treatment of later generations, which technological risk hinders. ${ }^{6}$ As a result, the structure of social discounting is endogenous and depends on technology, on the resolution of uncertainty over time, and on the planner's aversion to intrinsic risk. Among the special cases: discounting is exponential when the distribution problem is risk-less, i.e. the technology is uniform across histories; it is quasi-hyperbolic if all the risk resolves after the first period. ${ }^{7}$

Finally, the criterion avoids drastic and inadequate policy recommendations in the presence of fat-tailed catastrophic events (see Weitzman (2009) and the recent

\footnotetext{
${ }^{5}$ The recent literature on intergenerational social welfare with risk has proposed different alternatives that achieve this goal, including Traeger (2012), building on Kreps and Porteus (1978)'s recursive expected utility model, and Fleurbaey and Zuber (2014b), characterizing an ex-post criterion related to Fleurbaey (2010). Extensions to settings in which generations face a different probability of coming into existence include Asheim and Zuber (2013); Fleurbaey and Zuber (2014a); closely related, in the Online Appendix C, I allow for extinction and show how this can be used to characterize the pure time discount rate.

${ }^{6}$ The importance of the time resolution of risk is long known in the literature (Arrow and Fisher (1974); Hammitt et al. (1992); Hanemann (1989); Henry (1974); Pindyck (2000)), but is generally not accounted for in the evaluation of intergenerational risk.

${ }^{7}$ For a formalization of quasi-hyperbolic discounting see Laibson (1997). Non-exponential discounting is known to lead to the problem of time inconsistency (see Koopmans (1960)). Nevertheless, time varying discounting seems necessary to combine reasonable short-term discount factors with sensitivity to the long-run effects of climate change (Karp (2005), Gerlagh and Liski (2013)). Furthermore, time inconsistency is proven to be unavoidable when aggregating heterogeneous opinions over the "correct" discount factors (Weitzman, 2001) or when aggregating individuals with different discount factors (Zuber (2011), Jackson and Yariv (2014)). The design of optimal policies for time inconsistent discounters is addressed in Harstad (2013).
} 
discussions by Nordhaus (2011), Weitzman (2011), Stern (2013), and Millner (2013)). The intuition is that, according to the fair intergenerational utilitarian criterion, the more costly it is to transfer resources to a generation at a specific history, the lower is its legitimate claim to consumption (identified by the suggested fair prospect). Thus, even though the marginal well-being might be very high, such low levels of consumptions might be justified when, as in catastrophic scenarios, the marginal social cost of transferring resources to increase this low consumption were extremely high.

The characterized family of criteria thus avoids known drawbacks of discounted expected utilitarianism. The latter (i) cannot disentangle the social aversion to intergenerational inequality from the social aversion to risk (see Anthoff et al. (2009); Dasgupta (2008); Fleurbaey and Zuber (2014a)), (ii) is independent of the time resolution of risk (Pindyck (2000)), and (iii) might lead to ethically indefensible policy guidance in the presence of fat-tailed catastrophic events (Weitzman (2009)).

The remainder of the paper is organized as follows. In Section 2 and 3, I introduce the model and the axioms. In Section 4, I derive the characterization result. In Section 5, I defend a specific reference prospect and discuss its appeal in relation to the literature. In Sections 6 and 7, I present the consequences of the ranking in terms of discounting and fat-tailed catastrophic risks. I briefly conclude in Section 8.

\section{DECISION TREES AND RISKY PROSPECTS}

2.1. The model. Time is discrete and finite: $t \in T \equiv\{0, \ldots, \bar{t}\}$ with $0<\bar{t}<\infty$. Let $H$ denote the set of possible histories $h$, which can arise with strictly positive probability. A node $n$ is uniquely identified by the time $t \in T$ and by the set of histories $H(n) \subseteq H$ that are still possible at $n$. The set of nodes constitutes the event tree $N ; N_{t} \subseteq N$ is the subset of nodes at $t \in T ; N(n) \subseteq N$ denotes the set of nodes which succeed $n$, including $n$ itself. Let $n=0$ be the unique initial node, at $t=0$ (thus, $H(0)=H$ ). As usual, later partitions of histories are finer; i.e. for each $t \in T, n \in N_{t}$ and $n^{\prime} \in N_{t+1}$, either $H(n) \supseteq H\left(n^{\prime}\right)$ or $H(n) \bigcap H\left(n^{\prime}\right)=\emptyset$.

Let a decision tree associated to the event tree $N$ be denoted by:

$$
D \equiv\left\langle\left\langle\pi_{n}, F_{n}\right\rangle_{n \in N}, \underline{k}\right\rangle
$$

Each element of $D$ is defined as follows. For each period $t \in T$ and each node $n \in N_{t}$ : $\pi_{n} \in(0,1]$ is the compound probability that node $n$ is reached from node $0 ; F_{n}$ is the production function that transforms input $k_{n} \in \mathbb{R}_{+}$into output $y_{n} \in \mathbb{R}_{+}$. Let $\mathcal{F}$ be the 
set of all production functions $F: \mathbb{R}_{+} \rightarrow \mathbb{R}_{+}$that are continuous, strictly increasing, and satisfy no-free lunch; then, $F_{n} \in \mathcal{F}$. Output $y_{n}$ is either saved -let $s_{n} \in \mathbb{R}_{+}$ denote saving at $n$ - or assigned to the currently living generation -let $c_{n} \in \mathbb{R}_{+}$denote the outcome at $n$. Savings determine the capital stock of the immediate successor nodes, i.e. $k_{n^{\prime}}=s_{n}$ for each $n^{\prime} \in N_{+1}(n)$. The initial capital level is $\underline{k}>0$. A history $h \in H$ is then a specification of production technology at each period $t \in T$.

For each $t \in T$ and each $n \in N_{t}$, the conditional generations at $\mathbf{n}$ is the representative agent living at $t$ when node $n$ is reached. Each conditional generation is assumed to be born after the technology at $t$ is realized, so that the risk is exclusively faced by the ethical observer.

Let $\mathcal{D}$ be the domain of decision trees satisfying the above assumptions. For each $D \in \mathcal{D}$, a (risky) prospect for $\mathbf{D}$ is denoted by $c \equiv\left(\left\{c_{n}\right\}_{n \in N}\right) \in C(D) \equiv \mathbb{R}_{+}^{|N|}$. The set of feasible prospects for $\mathbf{D}$ is $C^{F}(D) \subset C(D)$ such that $c \in C^{F}(D)$ if for each $n \in N$ : (i) $y_{n}=F_{n}\left(k_{n}\right)$; (ii) $c_{n}+s_{n} \leq y_{n}$; (iii) $k_{n^{\prime}}=s_{n}$ for each $n^{\prime} \in N_{+1}(n)$; (iv) $k_{0}=\underline{k}$.

2.2. An illustration. The simplest version of the model consists of a two-period decision tree with two possible histories. Histories specify the second-period technology, which can be either $F_{G}$, with probability $\pi_{G}$, or $F_{B}$, with probability $\pi_{B}=1-\pi_{G}$. The production function at 0 is $F_{0}\left(k_{0}\right)$, represented in the left side of Fig.2.1: it is a function that maps capital stock $k_{0}$ into output $y_{0}$. Given the initial capital stock $\underline{k}$, the output available at 0 is $y_{0}^{*}$. In the right side of Fig.2.1, the production functions $F_{G}\left(s_{0}\right)$ and $F_{B}\left(s_{0}\right)$ describe how much output is produced at each possible history $G$ and $B$ for each saving decision $s_{0} \leq y_{0}^{*}$. For savings $s_{0}^{*}$, for example, the maximal consumption of generation 0 is $c_{0}^{*} \equiv y_{0}^{*}-s_{0}^{*}$, while conditional generations at $G$ and $B$ can consume $c_{G}^{*} \equiv F_{G}\left(s_{0}^{*}\right)$ and $c_{B}^{*} \equiv F_{B}\left(s_{0}^{*}\right)$ respectively.

It is worth highlighting two important aspects of the model. First, it is generally not possible to perfectly ensure against future technological risks and together distribute resources efficiently: the consumption possibilities at histories $G$ and $B$ are jointly determined by the saving $s_{0}$ and no policy can reduce the difference between $F_{G}\left(s_{0}\right)$ and $F_{B}\left(s_{0}\right)$. Second, the technology is "smooth": at each node (i) consumption can vary from zero to exhausting all resources available at that history and (ii) reducing consumption and increasing savings allows assigning (continuously) more consumption to all later generations. This characteristics of the model guarantees compactness of the consumption possibility set and, as discussed in Section 5, the existence of an equitable and non-wasteful prospect. 


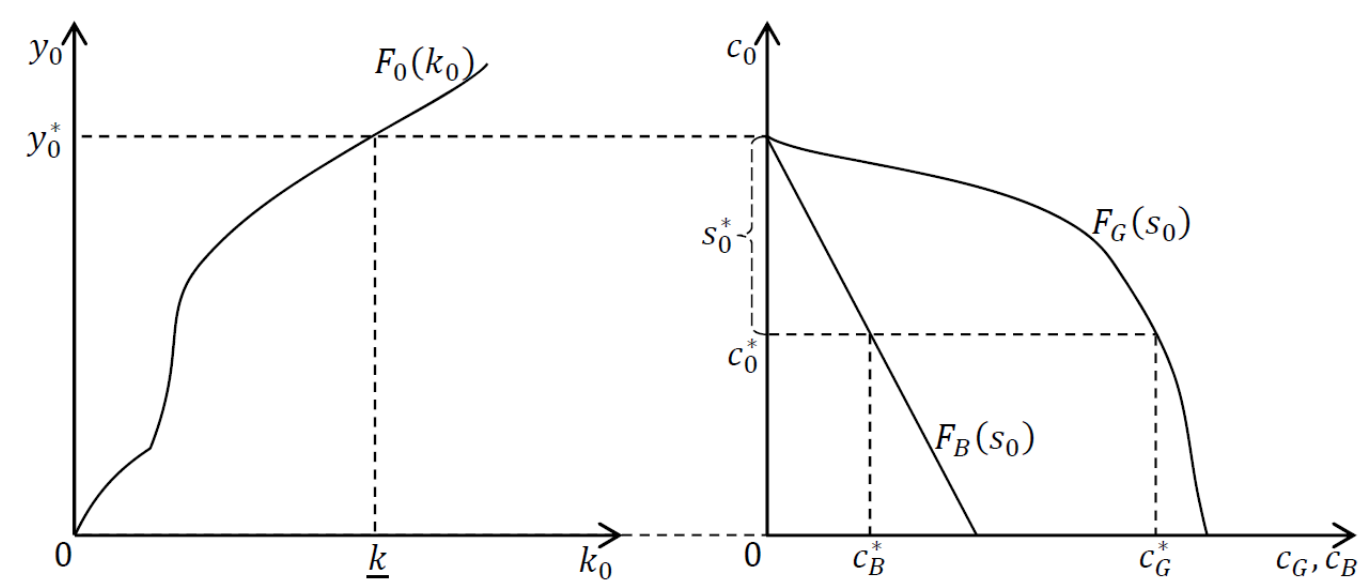

Figure 2.1. A two-period two-history model.

\section{The Axioms}

A social ordering of $\mathbf{D}$ is a complete and transitive binary relation defined over $C(D)$. A social ordering function $\succsim$ assigns to each decision tree $D \in \mathcal{D}$ a social ordering of $D$, denoted $\succsim_{D}{ }^{8}$ Thus, $c \succsim_{D} c^{\prime}$ means that the prospect $c$ is socially at least as desirable as $c^{\prime}$ for decision tree $D$. The symmetric and asymmetric counterparts of $\succsim_{D}$ are $\sim_{D}$ and $\succ_{D}$.

For the sake of applicability of the results to climate change models, I shall restrict the attention to social orderings that admit a continuous and separable welfare representation. Formally, for each $D \in \mathcal{D}$, there exist continuous functions $u_{n}$, one for each $n \in N$, and continuous functions $q_{t}$, one for each $t \in T$, such that the social ordering $\succsim_{D}$ is ordinally equivalent to:

$$
V(c ; D)=\sum_{t \in T} q_{t}\left(\sum_{n \in N_{t}} u_{n}\left(c_{n}\right)\right) .
$$

It is worthwhile stressing that this formulation is rather general: it does not require any anonymity across generations or histories, nor does it introduce monotonicity, discounting or probability weighting. This formulation rules out rankings that give full-priority to a subset of generations/histories like maximin, leximin, or evaluations related to the precautionary principle, but these can (and will) arise as limit cases. As shown in Appendix A, this functional form can be obtained from three independent

${ }^{8}$ The social ordering function approach has been successfully applied in the social choice literature to deal with non-comparable information about preferences: among the main advantages, it adds the flexibility of a complete ranking to the ethical appeal of fair allocation theory (see Fleurbaey and Maniquet (2011)). 
axioms: continuity, a separability condition across generations, and a separability condition across possible histories.

The first axiom is standard. A prospect is strictly preferred if it assigns at least as much consumption to each generation at each history, and strictly more to some.

Strict monotonicity. For each $D \in \mathcal{D}$ and each pair $c, \bar{c} \in C(D)$, if $c_{n} \geq \bar{c}_{n}$ for each $n \in N$ and $c_{n}>\bar{c}_{n}$ for some $n \in N$, then $c \succ_{D} \bar{c}$.

The ethical concern for intergenerational equity is introduced as a Pigou-Dalton transfer axiom (Pigou (1912); Dalton (1920)). In the original version of the axiom, a progressive transfer from a richer to a poorer person is welfare improving. The idea is that, provided the richer/poorer relation is not inverted, the transfer reduces inequality; thus, the after-transfer allocation is at least as desirable as the starting one. I shall introduce three differences.

First, who is to be considered rich or poor depends on what each conditional generation should be equitably assigned. Let the fair prospect $x \equiv\left(\left\{x_{n}\right\}_{n \in N}\right)$ be the most appealing way of distributing resources in the eyes of an egalitarian observer; the fair prospect is assumed to be uniquely selected by an allocation rule $\psi: \mathcal{D} \rightarrow C^{F}(\mathcal{D})$ and to assign a strictly positive consumption to each conditional generations, i.e. $\psi(D) \gg 0$ for each $D \in \mathcal{D} .^{9}$ It is natural to let this egalitarian reference be a constant and efficient distribution of consumptions; however, as this prospect might not be feasible, a trade-off between equity and efficiency arises. Since the characterization result is going to be independent of the way the fair prospect is determined, I shall postpone to Section 5 the discussion of its fairness properties.

Then, one generation is considered richer than another if at each possible history (i) the first is assigned a larger consumption than at the fair prospect and (ii) the second one is assigned a smaller consumption than at the fair prospect. ${ }^{10}$

Second, the transfer from the richer donor to the poorer recipient needs to be uniform across possible histories.

Third, the transfer is discounted at a factor $\beta \in(0,1]$. This parameter interprets the pure time preference of the ethical observer: for example, a transfer from a richer future generation $t$ to the poorer generation 0 is welfare increasing if the latter is given at least a fraction $\beta^{t}$ of the transferred resources. The characterization result is independent of the ethical view about the pure time preference $\beta$; a possible ethical

\footnotetext{
${ }^{9}$ For a recent overview of fair allocation theory, see Thomson (2011).

${ }^{10}$ The axiom remains silent about transfers in which both generations are better or worse than at the fair prospect; this makes the axiom considerably weaker than the original one.
} 
justification is proposed in the Online Appendix C, where the model is extended to account for the probability of extinction.

Intergenerational equity. For each $D \in \mathcal{D}$, each $x=\psi(D)$, each pair $c, \bar{c} \in C(D)$, each pair $t, t^{\prime} \in T$, and each $\delta \in \mathbb{R}_{+}$, if

(i) $c_{n}=\bar{c}_{n}-\frac{\delta}{\beta^{t}} \geq x_{n}$ for each $n \in N_{t}$;

(ii) $c_{n^{\prime}}=\bar{c}_{n^{\prime}}+\frac{\delta}{\beta^{t^{\prime}}} \leq x_{n^{\prime}}$ for each $n^{\prime} \in N_{t^{\prime}}$;

(iii) $c_{n^{\prime \prime}}=\bar{c}_{n^{\prime \prime}}$ for each $n^{\prime \prime} \in N \backslash\left\{N_{t} \bigcup N_{t^{\prime}}\right\}$,

then $c \succsim_{D} \bar{c}$.

The axiom reads as follows. Take two generations $t, t^{\prime} \in T$. At $\bar{c}, t$ is assigned more than at the fair prospect $x$ in each possible history (condition $i$ ); $t^{\prime}$ is assigned less than at the fair prospect $x$ in each possible history (condition $i i$ ). Define a timediscounted transfer $\delta$ from $t$ to $t^{\prime}$, uniform across possible histories, such that the first generation remains richer than the second also after the transfer. Then, ceteris paribus (condition $\mathrm{iii}$ ), the after-transfer prospect $c$ is socially weakly more desirable than the initial one $\bar{c}$.

The ethical concern for risk is related to the mean preserving spread (Rothschild and Stiglitz (1970)), suggesting that among equal-mean lotteries the ethical observer ought to choose the one with lowest risk. The mean preserving spread is obtained by transferring probability mass to the tales of the distribution, but can be equivalently expressed as a regressive transfer across histories, weighted by the likelihood of each.

The main difference is the introduction of the fair prospect to determine which equal-mean transfers lead to ethically more appealing prospects. In fact, consistent with the view that the fair prospect incorporates some notion of fairness, the meanpreserving transfer is considered welfare improving if it reduces the gap with such reference. More precisely, a prospect is socially more desirable if it is obtained through a mean-preserving transfer from a possible history where a generation is assigned more than at the fair prospect to another possible history where the same generation is assigned less than at the fair prospect.

Risk balancing. For each $D \in \mathcal{D}$, each $x=\psi(D)$, each pair $c, \bar{c} \in C(D)$, each $t \in T$, each pair $n, n^{\prime} \in N_{t}$, and each $\delta \in \mathbb{R}_{+}$, if

(i) $c_{n}=\bar{c}_{n}-\frac{\delta}{\pi_{n}} \geq x_{n}$;

(ii) $c_{n^{\prime}}=\bar{c}_{n^{\prime}}+\frac{\delta}{\pi_{n^{\prime}}} \leq x_{n^{\prime}}$;

(iii) $c_{n^{\prime \prime}}=\bar{c}_{n^{\prime \prime}}$ for each $n^{\prime \prime} \neq n, n^{\prime}$,

then $c \succ_{D} \bar{c}$. 
The axiom reads as follows. Consider a generation $t \in T$ and the prospect $\bar{c} \in$ $C(D)$. At node $n \in N_{t}$, the conditional generation is assigned more than at the fair prospect (condition $i$ ); at node $n^{\prime} \in N_{t}$, the conditional generation is instead assigned less than at the fair prospect (condition $i i$ ). Define a transfer from $n$ to $n^{\prime}$, weighted by the probability of each node, such that conditional generation $n$ remains richer than $n^{\prime}$ also after the transfer (in relative terms with respect to the fair prospect). Then, ceteris paribus (condition iii), the after-transfer prospect $c$ is strictly more desirable than the initial one $\bar{c}$.

The last axiom requires the ranking to be invariant to ratio-scale changes of the fair prospect. This equitable reference enters the axioms of intergenerational equity and risk balancing to establish the richer-poorer relation and the direction of a welfare improving transfer. Thus, requiring invariance of the social ordering with respect to proportional changes of the fair prospect is equivalent to demanding the richer-poorer relation to be a relative property and not absolute. ${ }^{11}$

Proportionality. For each pair $D, D^{\prime} \in \mathcal{D}$ such that the fair prospect $\psi(D)$ is proportional to the fair prospect $\psi\left(D^{\prime}\right)$, and for each pair $c, \bar{c} \in C(D), c \succsim_{D} \bar{c}$ if and only if $c \succsim_{D^{\prime}} \bar{c}$.

\section{The CHARACTERIZATION RESUlT}

I first define the social ordering. Let $D \in \mathcal{D}$ and $x \equiv \psi(D)$. For each generation $t \in T$, let the risk-adjusted discount factor be:

$$
\tilde{\beta}_{t} \equiv \beta^{t}\left(\frac{\sum_{n \in N_{t}} \pi_{n} x_{n}}{x_{0}}\right)
$$

It consists of two components: a pure time discounting part $\beta^{t}$ and a risk-adjustment factor $\left(\frac{\sum_{n \in N_{t}} \pi_{n} x_{n}}{x_{0}}\right)$, given by the average consumption assigned at the fair prospect to generation $t$ compared to generation 0 . Let generation t's welfare be measured by the function $v_{t}$ with the following functional form:

$$
v_{t}(c ; D) \equiv\left\{\begin{array}{cc}
\left(\sum_{n \in N_{t}} \frac{\pi_{n} x_{n}}{\sum_{\bar{n} \in N_{t}} \pi_{\bar{n} x_{\bar{n}}}}\left(\frac{c_{n}}{x_{n}}\right)^{\gamma}\right)^{\frac{1}{\gamma}} & \gamma<1, \gamma \neq 0 \\
\prod_{n \in N_{t}}\left(\frac{c_{n}}{x_{n}}\right)^{\sum_{\bar{n} \in x_{n}} \pi_{\bar{n}} x_{\bar{n}}} & \gamma=0
\end{array}\right.
$$

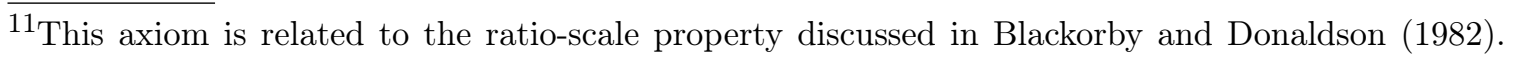
The first difference is that their axiom is an informational requirement on comparable utilities. The second difference is that ratio-scale is here not imposed on the vectors of consumptions to be ranked -object of social comparison- but on the reference prospect, introduced through the fairness axioms.
} 
Fair intergenerational utilitarianism: For each $D \in \mathcal{D}$,

$$
V(c ; D) \equiv\left\{\begin{array}{cc}
\sum_{t \in T} \tilde{\beta}_{t} \frac{\left(v_{t}(c ; D)\right)^{1-\rho}}{1-\rho} & \rho \geq 0, \rho \neq 1 \\
\sum_{t \in T} \tilde{\beta}_{t} \ln \left(v_{t}(c ; D)\right) & \rho=1
\end{array}\right.
$$

where, for each $t \in T, \tilde{\beta}_{t}$ is defined in (4.1) and $v_{t}(c ; D)$ in (4.2).

Theorem 1. On the domain $\mathcal{D}$, the following statements are equivalent:

$S 1$ : $\quad$ the social ordering function $\succsim$ satisfies strict monotonicity, intergenerational equity, risk-balancing, and proportionality;

$S 2: \quad$ for each $D \in \mathcal{D}$, the social ordering $\succsim_{D}$ can be represented by the fair intergenerational utilitarian criterion.

The way the axioms contribute to the result is as follows. Equity across time and histories, both expressed as transfer principles with respect to the reference prospect, force social welfare to be a function of the relative size of the assigned consumptions with respect to the fair prospect. As a consequence, invariance to proportional changes of the fair prospect plays a similar role to invariance with respect to ratio-scale changes of assigned consumption and the power representation should not be too surprising (see Atkinson, 1970; Blackorby and Donaldson, 1982). What is perhaps less obvious is how the nested time/history aggregation structure of the welfare representation cuts out an additional role to the fair prospect: (i) in terms of weighting relative consumptions in each generation's welfare (4.2); and (ii) in terms of discounting each generation's welfare (4.1).

Before discussing the choice of the fair prospect and its consequences for social welfare, few general remarks are in order.

Remark 1. Ethical considerations for risk enter the criterion twice: first, in the choice of the fair prospect; second, in the evaluation of the deviations from this reference. This allows disentangling two corresponding sources of risk. Intrinsic risk is determined by the resource distribution problem and is unavoidable even at the egalitarian prospect $x$ due to the non-insurable technological risk. I shall discuss further this source of risk in the next section, where the allocation rule singling out this equitable reference is characterized. Option risk is incurred in only if the planner "decides" to deviate from the fair prospect $x$ to achieve a higher social welfare by redistributing consumption differently across histories; aversion to such risk is captured by the elasticity parameter $\gamma$ in the definition of each generation's welfare (4.2). 
Remark 2. At the limit for $\gamma \rightarrow 1$ and $\rho=0$, the fair intergenerational utilitarian criterion is discounted expected utilitarian. From (4.2), it is immediate that when $\gamma \rightarrow 1$, the welfare of each generation is the average consumption (note that the denominator $\sum_{\bar{n} \in N_{t}} \pi_{\bar{n}} x_{\bar{n}}$ cancels out with the corresponding term in the risk-adjusted discount factor). Then, $\rho=0$ implies by (4.3) that each discounted generation's welfare is additively aggregated. As a result, social welfare is $V(c, D)=\sum_{t \in T} \beta^{t}\left(\sum_{n \in N_{t}} \pi_{n} c_{n}\right)$ and expected utilitarianism is recovered.

Remark 3. At the limit for infinite aversion to option risk $(\gamma \rightarrow-\infty)$ and infinite aversion to intergenerational inequality $(\rho \rightarrow \infty)$, the fair intergenerational utilitarian criterion is a maximin type of ranking. From (4.2) and (4.3), when $\gamma_{t} \rightarrow-\infty$ and $\rho \rightarrow$ $\infty$, the welfare of society is determined by the node with the smallest consumption relative to the fair prospect: $V(c, D)=\min _{n \in N} \frac{c_{n}}{x_{n}}$. The fact that the highest ranked prospect is the fair prospect further emphasizes its role as the egalitarian way of distributing resources.

Remark 4. When $\gamma=1-\rho, V(c, D)=\sum_{t \in T} \beta^{t}\left(\sum_{n \in N_{t}} \pi_{n} \frac{c_{n}^{1-\rho} x_{n}^{\rho}}{1-\rho}\right)$. In this case, the planner values equally proportional deviations from the fair prospect that redistribute resources over time (after correcting for the discount factor $\beta$ ) and deviations from the fair prospect that redistribute resources across possible histories (after correcting for the probability of each).

\section{The Fair Prospect}

5.1. The fair rule: a characterization. Let a rule $\phi$ be a correspondence that associates to each decision tree $D \in \mathcal{D}$ a non-empty subset of feasible prospects $\phi(D) \subseteq C^{F}(D)$. The uniqueness of the selection, assumed in Section 3 with allocation rule $\psi$, is here a result of the fairness properties to be introduced next.

The first property guarantees that the selected prospect $x$ assigns all the resources available in the economy: any larger prospect $x^{\prime}>x$ is not feasible.

Maximality. For each $D \in \mathcal{D}, x \in \phi(D)$ is such that:

$$
x^{\prime}>x \Longrightarrow x^{\prime} \notin C^{F}(D) .
$$

The second property requires each consumption/saving choice to treat fairly all the affected conditional generations. At each node $n$, the part of output $y_{n}$ leftover for later use, i.e. $s_{n}$, should be such that the consumption of the current conditional generation $x_{n}$ be considered socially as desirable as the risky consumption assigned to the later conditional generations (at histories that are still possible at $n$ ). For the 
example in Fig. 2.1, a prospect $\left(c_{0}^{*}, c_{G}^{*}, c_{B}^{*}\right)$ is considered equitable if $c_{0}^{*}$ is socially as desirable as a lottery that assigns $c_{G}^{*}$ with probability $\pi_{G}$ and $c_{B}^{*}$ with probability $\pi_{B} \cdot{ }^{12}$

The comparison between a certain consumption and a consumption lottery is expressed in terms of the quasi-linear mean (first characterized by De Finetti (1931) and Hardy et al. (1934) and later named "equally-distributed equivalent" by Atkinson (1970)). ${ }^{13}$ Formally, let $\mu: \mathbb{R} \rightarrow \mathbb{R}$ be a continuous, strictly increasing, and concave function that measures the risk aversion at the fair prospect or, as defined above, aversion to intrinsic risk.

Interim egalitarianism. For each $D \in \mathcal{D}, x \in \phi(D)$ is such that for each $t \in$ $[0, \bar{t}-1]$, each $n \in N_{t}$, and each $t^{\prime}>t$ :

$$
x_{n}=\mu^{-1}\left[\sum_{n^{\prime} \in N_{t^{\prime}}(n)} \frac{\pi_{n^{\prime}}}{\sum_{n^{\prime \prime} \in N_{t^{\prime}}(n)} \pi_{n^{\prime \prime}}} \mu\left(x_{n^{\prime}}\right)\right] .
$$

Define the fair rule $\phi^{*}$ as the one that satisfies maximality and interim egalitarianism. Then, the following result shows that the rule is well-defined.

Proposition 1. The fair rule $\phi^{*}$ associates to each decision tree $D \in \mathcal{D}$ a unique fair prospect $\left\{x^{*}\right\} \equiv \phi^{*}(D)$.

Proof. Let $D \in \mathcal{D}$. Let $C^{F I E} \subset C^{F}(D)$ be the subset of feasible prospects satisfying interim egalitarianism. Let $C_{0} \equiv\left\{\bar{c}_{0} \in \mathbb{R}_{+} \mid c_{0}=\bar{c}_{0}\right.$ for some $\left.c \in C^{F I E}\right\}$. The set $C_{0}$ is non-empty: since $c=0 \in C^{F I E}, 0 \in C_{0}$. The set $C_{0}$ is bounded from above: there is no $c \in C^{F}(D)$ such that $c_{0}>F_{0}(\underline{k})$. The set $C_{0}$ is compact: this follows from the continuity of the production functions. Let $c^{*} \in C^{F I E}$ be such that $c_{0}^{*}$ is the maximal element of $C_{0}$. By construction it satisfies interim egalitarianism. By contradiction, assume that $c^{*}$ is not maximal: there exists $c^{\prime} \in C^{F}(D)$ such that $c_{n}^{\prime} \geq c_{n}^{*}$ for each $n \in N$ and $c_{n}^{\prime}>c_{n}^{*}$ for some $n \in N$. Since technology is continuous, strictly increasing, and satisfies no free lunch, there exists a $c^{\prime \prime} \in C^{F I E}$ such that $c^{\prime \prime} \gg c^{*}$, contradicting $c_{0}^{*}$ being a maximal element of $C_{0}$. This implies that $\phi^{*}(D) \neq \emptyset$.

By contradiction, assume for some $D \in \mathcal{D}, \phi^{*}(D)$ is not a singleton, i.e. there exist a pair $c, \bar{c} \in \phi^{*}(D)$ with $c \neq \bar{c}$. Let $t \in T$ be the first period for which

\footnotetext{
${ }^{12} \mathrm{~A}$ related recursive way to evaluate risky prospects is studied by Asheim and Brekke (2002) to define sustainability in a setting with stochastic capital management.

${ }^{13}$ More general alternatives can be introduced to define the certainty equivalent of a lottery: the quasi-linear mean is however the only one that displays the ratio-rescaling invariance property (see Bossert and Weymark (2004)). In a one-shot risk setting, a family of related criteria has also been characterized by Fleurbaey (2010); Grant et al. (2012); Fleurbaey and Zuber (2014a): welfare is measured by the expected value of the equally-distributed equivalent evaluation of the outcomes at each state of nature.
} 
$c_{n} \neq \bar{c}_{n}$ for some $n \in N_{t}$. If $t=0, c_{0} \gtrless \bar{c}_{0}$ and the same argument as above leads to a contradiction of maximality. If $t>0$, since earlier production and consumptions are equal, it must be true that for each $n \in N_{t}, k_{n}=\bar{k}_{n}$. For each $n \in N_{t}$, let $C_{n}^{F I E} \equiv\left\{c^{\prime} \in C^{F I E} \mid c_{n^{\prime}}^{\prime}=c_{n^{\prime}}=\bar{c}_{n^{\prime}}\right.$ for all $n^{\prime} \in N_{t^{\prime}}$ with $\left.t^{\prime}<t\right\}$ and $C_{n} \equiv$ $\left\{c_{n}^{\prime} \in \mathbb{R}_{+} \mid c_{n}^{\prime \prime}=c_{n}^{\prime}\right.$ for some $\left.c^{\prime \prime} \in C_{n}^{F I E}\right\}$. The same reasoning as for $C_{0}$ proves that $c_{n}=\bar{c}_{n}$ and shows that the fair rule $\phi^{*}$ selects a unique prospect for each decision tree in the domain.

Before moving to a discussion of interim egalitarianism with respect to the literature, I shall highlight that the fair allocation rule $\phi^{*}$ guarantees that each conditional generation be assigned a strictly positive consumption, as assumed for the characterization result.

Corollary 1. For each $D \in \mathcal{D}$, the fair prospect $\left\{x^{*}\right\} \equiv \phi^{*}(D)$ is such that $x_{n}^{*}>0$ for each $n \in N$.

Proof. By contradiction assume that for some $D \in \mathcal{D},\left\{x^{*}\right\} \equiv \phi^{*}(D), t \in T$, and $n \in N_{t}$, it holds that $x_{n}^{*}=0$. By definition of interim egalitarianism (and $x_{\bar{n}}^{*} \geq 0$ for each $\bar{n} \in N)$, it follows that $x_{n^{\prime}}^{*}=0$ for each $n^{\prime} \in N(n)$. By feasibility of $x^{*}, k_{n} \geq 0$. If $k_{n}>0, F_{n}\left(k_{n}\right)>0$ by strict monotonicity and no free lunch of the production functions. Then, there would exist $\bar{x}>x^{*}$ with $\bar{x} \in C^{F}(D)$ contradicting maximality. Thus, $k_{n}=0$. By feasibility, this implies that $k_{n^{\prime}}=0$ for each $n^{\prime} \in N_{t}\left(n^{-}\right.$) (all nodes with the same predecessor as $n$ ) and, by no free lunch, $x_{n^{\prime}}^{*}=0$ for each $n^{\prime} \in N\left(n^{-1}\right) \backslash n^{-1}$. By interim egalitarianism, it follows that $x_{n^{-1}}^{*}=0$. Since $x_{n}^{*}=0$ implies that $x_{n^{-}}^{*}=0$ and that $x_{n^{\prime}}^{*}=0$ for each $n^{\prime} \in N\left(n^{-}\right)$, a reasoning by induction leads to $x^{*}=0$. This contradicts maximality (since $\underline{k}>0$ and $F_{0}(\underline{k})>0$ ) and proves the result.

The positivity of the egalitarian reference is ethically appealing, also in light of the existence of feasible prospects that assign to each conditional generation a positive consumption level. However, this property rules out reference prospects obtained by maximizing the expected equally distributed equivalent social welfare function proposed by Fleurbaey (2010) and Grant et al. (2012): when the technology of a period is (at each possible history) sufficiently more productive than at other periods, it will be optimal to assign a positive consumption to that conditional generations only (unless the equally distributed equivalent exhibits infinite degree of inequality aversion). The reason is that social welfare is measured as the weighted average of the constant consumption deemed as desirable as the consumption stream assigned 
at each history; it is thus indifferent to permutations of consumptions across time and allows for all consumption to be assigned to only one generation.

Another alternative to the fair rule $\phi^{*}$ is to choose a constant prospect, i.e. a prospect that assigns to each conditional generation the same consumption. This case is discussed in Section 6 , as it arises as a special case of $\phi^{*}$, for a subdomain of decision trees.

5.2. Ex-ante, ex-post, and interim egalitarianism. In this subsection, I further discuss the appeal of interim egalitarianism in relation to the literature.

The usual representation of one-shot risky social situations takes a matrix form. Let each column describe the lottery assigned to an agent and each row describe the social distribution for a specific state of nature. The following one-shot prospects $(I)$, $(I I)$, and $(I I I)$ are examples of risky social situations involving two agents (1 and 2) and two equally-likely states of nature $\left(\sigma_{1}\right.$ and $\left.\sigma_{2}\right)$.

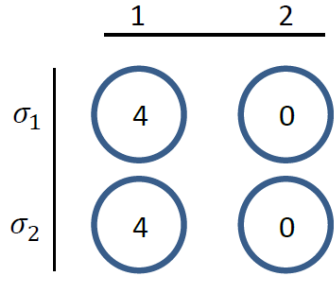

$(I)$

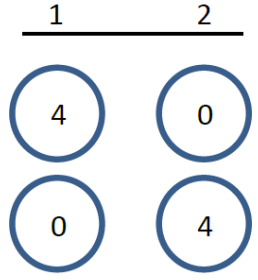

$(I I)$

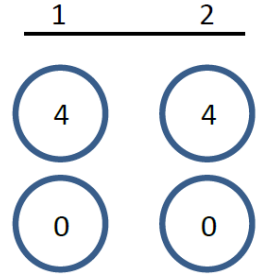

$(I I I)$

According to the expected utilitarian criterion, these are all equally desirable. ${ }^{14}$ This indifference is not universally accepted. Diamond (1967) suggests that prospect $(I I)$ should be considered socially more desirable than $(I)$, as it gives both agents 1 and 2 "a fair shake": they are given an equal chance of achieving the high and low outcomes; in other words, their lotteries are ex-ante equivalent; this concern for fairness has been named ex-ante egalitarianism. More recently, Broome (1991) suggested that, while both prospect $(I I)$ and $(I I I)$ assign the same ex-ante prospect, the latter is the one to be preferred as it leads to more equitable distributions (whatever the realized state of nature is); this concern for distributional equity has been named ex-post egalitarianism.

Extending this reasoning to the present setting is not immediate. First, the interpretation of the arguments is different as generations do not have preferences over their assigned lottery: each conditional generations is assumed to be born after the

\footnotetext{
${ }^{14}$ Even if the consumption (or monetary outcome) is replaced by the corresponding utility level, the conclusion is not affected: the expected utilitarian criterion is invariant with respect to permuting these levels across agents and states of nature.
} 
realization of each period's technological shock. Second, the above prospects $(I I)$ and $(I I I)$ do not fit the structure of the studied multi-stage lotteries, since these require the outcome of the first generation to be equal across histories. ${ }^{15}$ I will argue that similar arguments for fairness and equity can be presented.

Consider an economy spanning three periods, i.e. $\{0,1,2\}$. Let the decision tree consist of two equally-likely histories, depending on whether the technology of periods 1 and 2 is of type $G$ or of type $B$. A prospect is represented by reporting the outcome assigned to each generation at each possible history in the corresponding node of the tree, as in the following examples $(\bar{I}),(\overline{I I})$, and $(\overline{I I I})$. Note that outcomes assigned at time 1 and 2 are lotteries since these can be conditioned on the information that is revealed by that time, i.e. the type of technology.

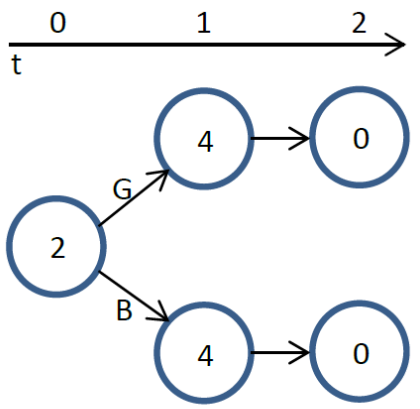

$(\bar{I})$

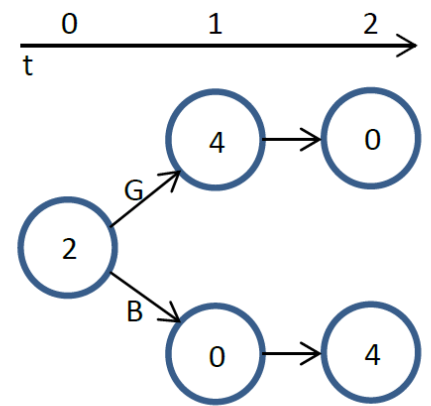

$(\overline{I I})$

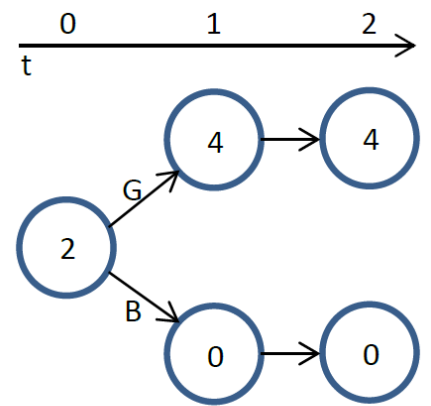

$(\overline{I I I})$

The (undiscounted) expected utilitarian criterion would rank these prospects equally. Stretching Diamond's argument, one can agree that prospect $(\overline{I I})$ is socially more attractive than $(\bar{I})$, as it impedes generation 1 to spoil generation 2 . As in the corresponding example with one-shot lotteries, prospects $(\overline{I I})$ and $(\overline{I I I})$ are ex-ante equivalent. Extending the concern for ex-post egalitarianism of Broome (1991), prospect $(\overline{I I I})$ is to be preferred to $(\overline{I I})$ since the distribution that would arise at each history is more equitable. ${ }^{16}$

\footnotetext{
${ }^{15}$ It is immediate that the tree structure of multi-stage lotteries does not allow to introduce wellknown axioms from the literature. In particular, equal consideration for all generations can not be based on anonymity as permuting their risky prospects is not well-defined: generations living at different times are substantially different with respect to how much risk is revealed and disregarding such difference (as in a one-shot lottery setting) is inappropriate to assess intergenerational risk. Risk is a particularly powerful argument against intergenerational anonymity, complementary to the one based on multidimensional commodity spaces raised by Piacquadio (2014).

${ }^{16}$ To see this, write the prospects in the classical matrix form and compare the outcomes, history by history, only in terms of inequality among the assigned outcomes. If the reader agrees that $(2,4,0)$ is less egalitarian than $(2,4,4)$ and $(2,0,4)$ is less egalitarian than $(2,0,0)$, the argument applies and leads to $(\overline{I I I})$ being better than $(\overline{I I})$.
} 
While I agree that $(\overline{I I I})$ is socially more desirable than $(\overline{I I})$, I shall present a different motivation that stems out from the decision-tree structure. In fact, when the outcome of each conditional generation at time 1 is to be set, the decision maker is informed whether the technology is $G$ or $B$. At prospect $(\overline{I I})$, period 1's saving decisions are known to lead to large inequalities in the assignments at 1 and 2: either the first gets 4 units and the second gets 0 or the reverse. Whereas, prospect $(\overline{I I I})$ guarantees distributional equity whether technology is $G$ (and both get 4 ) or $B$ (and both get 0$).{ }^{17}$

This reasoning motivates interim egalitarianism and justifies the fair prospect $\phi^{*}$. The idea is that ex-ante egalitarianism should hold each time a distributional decision is taken, based on the information that is available at that time. The requirement is clearly stronger than Diamond (1967)'s concern for ex-ante egalitarianism. More interestingly, the idea of conditioning ex-ante egalitarianism on the available information impedes to ethically justify unequal distribution based on outcomes at histories that cannot realize anymore (as in prospect $\overline{I I}$ ): it thus recursively incorporates a concern for ex-post egalitarianism.

\section{EXPONEntial, QUASI-HYPERBOLIC, AND HYPERBOliC DisCOUNTING}

In the next two sections, I shall discuss the implications of Theorem 1 when selecting the fair prospect based on the fair rule $\phi^{*}$ : fair intergenerational utilitarian criterion and fair prospect will refer to the fair rule $\phi^{*}$, even if not explicitly mentioned. This section is concerned with the structure of social discounting: the implications are presented in the form of corollaries.

The first one tells that as the planner becomes more averse to intrinsic risk (larger concavity of $\mu$ ), the fair prospect $x^{*}$ assigns more resources to future generations, and, as a consequence, also the ranking of alternatives gives more weight to future generations.

\footnotetext{
${ }^{17}$ A different viewpoint is defended by Traeger (2012): his "intertemporal risk averse decision maker" would prefer an alternating high/low outcomes $(\overline{I I})$ to the fair lottery between always high and always low outcomes $(\overline{I I I})$. The reason is that in Traeger the decision maker lives for the whole time horizon and discounts the future based on the intertemporal elasticity of substitution and the risk aversion; his policy implication is that (exogenously) increasing uncertainty leads to more discounting. Instead, the fair intergenerational utilitarian criterion compares one-period living generations, for which intergenerational equity requires assigning more weight to generations that face more uncertain resource distributions; thus more uncertainty leads to more weight to future generations.
} 
Corollary 2. For each $D \in \mathcal{D}$, let the social ordering $\succsim_{D}$ be representable by the fair intergenerational utilitarian criterion. Then, as $\mu$ becomes more concave, the discount factor $\tilde{\beta}_{t+1} / \tilde{\beta}_{t}$ increases.

Proof. By the definition of fair intergenerational criterion,

$$
\frac{\tilde{\beta}_{t+1}}{\tilde{\beta}_{t}}=\beta \frac{\sum_{n^{\prime} \in N_{t+1}} \pi_{n^{\prime}} x_{n^{\prime}}^{*}}{\sum_{n \in N_{t}} \pi_{n} x_{n}^{*}}
$$

By interim egalitarianism, $x_{n}^{*}=\mu^{-1}\left[\sum_{n^{\prime} \in N_{t+1}} \pi_{n^{\prime}} \mu\left(x_{n^{\prime}}^{*}\right)\right]$ for each $n \in N_{t}$. When the concavity of $\mu$ increases, also the ratio $\frac{\sum_{n^{\prime} \in N_{+1}(n)} \pi_{n^{\prime}} x_{n^{\prime}}^{*}}{x_{n}^{*}}$ does. Since $\sum_{n^{\prime} \in N_{t+1}} \pi_{n^{\prime}} x_{n^{\prime}}^{*}=$ $\sum_{n \in N_{t}} \pi_{n}\left[\sum_{n^{\prime} \in N_{+1}(n)} \pi_{n^{\prime}} x_{n^{\prime}}^{*}\right]$, the result obtains.

The form of the risk-adjusted discount factor depends on the interaction between (intrinsic) risk, its resolution over time, and the planner's aversion to it. The special case of exponential discounting arises when the fair prospect $x^{*}$ is constant over time. This happens when either the ethical observer is indifferent to intrinsic risk -i.e. when the planner is risk-neutral at the fair prospect ( $\mu$ is linear)- or when the resource distribution problem is risk-free - i.e. technology is uniform across histories. These cases are formalized in the next two corollaries.

Corollary 3. For each $D \in \mathcal{D}$, let the social ordering $\succsim_{D}$ be representable by the fair intergenerational utilitarian criterion. Then, if the planner is intrinsic-risk neutral ( $\mu$ is linear), discounting is exponential.

Proof. The formula of the risk-adjusted discount factor is given in (4.1). When $\mu$ is linear, $x_{n}^{*}=\sum_{n^{\prime} \in N_{+1}(n)} \pi_{n^{\prime}} x_{n^{\prime}}^{*}$ for each $n \in N$ and, as a consequence, $\tilde{\beta}_{t}=\beta^{t}$ for each $t \in T$.

Corollary 4. For each $D \in \mathcal{D}$, let the social ordering $\succsim_{D}$ be representable by the fair intergenerational utilitarian criterion. Then, whenever for each $t \in T$ and each pair $n, n^{\prime} \in N_{t}, F_{n}=F_{n^{\prime}}$, discounting is exponential.

Proof. When technology is equal across histories, the fair prospect $x^{*}$ is constant, i.e. $x_{n}^{*}=x_{n^{\prime}}^{*}$ for each $n, n^{\prime} \in N$. Then, the risk-adjusted discount factor in (4.1) simplifies and becomes $\tilde{\beta}_{t}=\beta^{t}$; which is the formulation of exponential discounting. 
When the fair prospect is constant, the fair intergenerational utilitarian criterion simplifies as follows:

$$
V(c ; D)=\sum_{t} \frac{\beta^{t}}{1-\rho}\left[\left(\sum_{n \in N_{t}} \pi_{n}\left(c_{n}\right)^{\gamma}\right)^{\frac{1}{\gamma}}\right]^{1-\rho} .
$$

This same functional form would result from the characterization for a different choice of equitable reference, i.e. one for which consumptions across time and possible histories are equalized independently of the technology. Although such criterion disentangles aversion to risk from aversion to intergenerational inequality, it is insensitive to the time disclosure of risk, it is insensitive to the social cost of transferring resources across possible histories, and is oversensitive to fat-tailed catastrophic events (see next section).

Another interesting case arises when risk resolves all-together in period 1 . Let $\mathcal{D}^{1} \subset$ $\mathcal{D}$ be the sub-domain of decision trees such that for each $n \in N_{1}$ and each $n^{\prime} \in N(n)$, $N_{+1}\left(n^{\prime}\right)$ is a singleton. The full evolution of technology becomes completely known as soon as the planner is in any node $n \in N_{1}$. Applying the interim egalitarianism condition of the fair rule $\phi^{*}$, this implies that the fair prospect $x^{*}$ remains constant from period 1 onwards, i.e. for each $n \in N_{1}$ and each $n^{\prime} \in N(n), x_{n}^{*}=x_{n^{\prime}}^{*}$ Thus, as the next corollary states, the fair intergenerational utilitarian criterion discounts the future in a quasi-hyperbolic way (Laibson (1997)).

Corollary 5. On the domain $\mathcal{D}^{1}$, if the social ordering ${ }_{D}$ can be represented by the fair intergenerational utilitarian criterion, then discounting is quasi-hyperbolic.

Proof. Let $\theta_{t} \equiv \sum_{n \in N_{t}} \pi_{n} x_{n}^{*}$. By the definition of fair rule and the domain restriction, $\frac{\theta_{t}}{x_{0}^{*}}=\frac{\theta_{t^{\prime}}}{x_{0}^{*}} \equiv \theta$ for each $t, t^{\prime} \geq 1$. Thus, the risk-adjusted discount factor in (4.1) is $\tilde{\beta}_{0}=\beta^{0}\left(\frac{x_{0}^{*}}{x_{0}^{*}}\right)=1$ for generation 0 and $\tilde{\beta}_{t}=\beta^{t}\left(\frac{\theta_{t}}{x_{0}}\right)=\beta^{t} \theta$ for each $t \geq 1$.

Finally, the case of hyperbolic discounting arises when the difference between the inverse of each consecutive risk-adjusted discount factors is a positive constant.

Corollary 6. For each $D \in \mathcal{D}$, let the social ordering $\succsim_{D}$ be representable by the fair intergenerational utilitarian criterion. Then, whenever the fair prospect $x^{*}$ is such that there exists $a \zeta>0$ with $\zeta=\frac{1}{\tilde{\beta}_{t+1}}-\frac{1}{\tilde{\beta}_{t}}$ for each $t \in[0, \bar{t}-1]$, then discounting is hyperbolic. 
Proof. By simple substitution, for each $t \in[0, \bar{t}-1]$, the discount factor between $t+1$ and $t$ is $\frac{\tilde{\beta}_{t+1}}{\tilde{\beta}_{t}}=1-\zeta \tilde{\beta}_{t+1}$. Since $\tilde{\beta}_{0} \equiv 1$, it follows that $\tilde{\beta}_{1}=\frac{1}{1+\zeta}$ and, by recursive substitution, that $\tilde{\beta}_{t}=\frac{1}{1+\zeta t}$ for each $t \in T$.

\section{FAT-TAiled CATASTROPHiC RISKS}

In this section, I discuss how the criterion based on $\phi^{*}$ performs when the resource allocation problem $D \in \mathcal{D}$ has the typical features of catastrophic fat-tailed risk. The "dismal theorem" states that the amount of resources to be saved to future agents is highly sensitive to catastrophic fat-tailed events. ${ }^{18}$ To exemplify the issue, consider a two-period two-history decision tree $D$ : in the second period technology can be good, $G$, with probability $\pi_{G}$, or bad, $B$, with probability $\pi_{B}=1-\pi_{G}$. Let initial capital be $\underline{k}=1$ and let technologies be as follows: $F_{0}\left(k_{0}\right)=k_{0} ; F_{G}\left(k_{G}\right)=k_{G}$; and $F_{B}\left(k_{B}\right)=\varepsilon k_{B}$ with $\varepsilon>0$. Clearly, $k_{G}=k_{B}=s_{0}$ where $s_{0}$ is the saving at node 0 . The idea is to analyze the intergenerational distribution problem for smaller and smaller values of $\varepsilon$, interpreted as history $B$ becoming more and more catastrophic.

The expected utilitarian planner maximizes a social welfare function $W^{e u}(c) \equiv$ $u\left(c_{0}\right)+\beta\left[\pi_{G} u\left(c_{G}\right)+\pi_{B} u\left(c_{B}\right)\right]$, where $u$ is a well-behaved utility function. The optimal policy satisfies the following (interior) first order condition:

$$
u^{\prime}\left(c_{0}^{*}\right)=\beta\left[\pi_{G} u^{\prime}\left(1-c_{0}^{*}\right)+\pi_{B} \varepsilon u^{\prime}\left(\varepsilon\left(1-c_{0}^{*}\right)\right)\right] .
$$

The absolute priority to future consumptions, consequence of the dismal theorem, can arise when the bad state becomes catastrophic: if $\lim _{c \rightarrow 0} u^{\prime}(c)=\infty$, then $\pi_{B} \varepsilon u^{\prime}\left(\varepsilon\left(1-c_{0}^{*}\right)\right)$ may tend to infinity as $\varepsilon \rightarrow 0$ (as in the case of a CRRA utility function with less than unitary elasticity of substitution).

Consider instead the maximization problem of a fair intergenerational utilitarian planner. Let $\tilde{\pi}_{G} \equiv \pi_{G} x_{G}^{*}$ and $\tilde{\pi}_{B} \equiv \pi_{B} x_{B}^{*}$. The welfare criterion is:

$$
V(c ; D)=\frac{\left(\frac{c_{0}}{x_{0}^{*}}\right)^{1-\rho}}{1-\rho}+\beta\left(\frac{\tilde{\pi}_{G}+\tilde{\pi}_{B}}{x_{0}^{*}}\right) \frac{\left(\frac{\tilde{\pi}_{G}}{\tilde{\pi}_{G}+\tilde{\pi}_{B}}\left(\frac{c_{G}}{x_{G}^{*}}\right)^{\gamma}+\frac{\tilde{\pi}_{B}}{\tilde{\pi}_{G}+\tilde{\pi}_{B}}\left(\frac{c_{B}}{x_{B}^{*}}\right)^{\gamma}\right)^{\frac{1-\rho}{\gamma}}}{1-\rho},
$$

where $\rho \geq 0(\rho \neq 1)$ measures the intergenerational inequality aversion and $\gamma<1$ $(\gamma \neq 0)$ measures the aversion to option risk. The optimal conditional allocation $c^{*}$

\footnotetext{
${ }^{18}$ See Weitzman (2009). This result has been largely criticized. The arguments opposed to the dismal theorem are reviewed and classified by Millner (2013) in 3 categories: the relevance of the dismal theorem for cost-benefit analysis; the correctness of fat-tailed probability distributions for modeling climate change events; and the behavioral/ethical foundation of the utility function. The present results contribute to the third category, by proposing an endogenous way to measure and compare utilities of consumptions.
} 
is such that:

$$
\left(\frac{c_{0}^{*}}{x_{0}^{*}}\right)^{-\rho}=\beta\left[\pi_{G}\left(\frac{1-c_{0}^{*}}{x_{G}^{*}}\right)^{\gamma-1}+\pi_{B} \varepsilon\left(\frac{\varepsilon\left(1-c_{0}^{*}\right)}{x_{B}^{*}}\right)^{\gamma-1}\right] \xi
$$

where $\xi=\left(\frac{\tilde{\pi}_{G}}{\tilde{\pi}_{G}+\tilde{\pi}_{B}}\left(\frac{1-c_{0}^{*}}{x_{G}^{*}}\right)^{\gamma}+\frac{\tilde{\pi}_{B}}{\tilde{\pi}_{G}+\tilde{\pi}_{B}}\left(\frac{\varepsilon\left(1-c_{0}^{*}\right)}{x_{B}^{*}}\right)^{\gamma}\right)^{\frac{1-\rho}{\gamma}-1}$.

At a first glance it seems that the dismal theorem applies: when $\gamma<0$ and the event becomes catastrophic $(\varepsilon \rightarrow 0), \pi_{B} \varepsilon\left(\varepsilon\left(1-c_{0}^{*}\right)\right)^{\gamma-1}$ tends to infinity. This conclusion is however wrong as the fair prospect $x^{*}$ is endogenously determined by the fair rule $\phi^{*}$ and depends on the technological parameter $\varepsilon$. By interim egalitarianism and maximality, $x^{*}$ is such that $\mu\left(x_{0}^{*}\right)=\pi_{G} \mu\left(1-x_{0}^{*}\right)+\pi_{B} \mu\left(\varepsilon\left(1-x_{0}^{*}\right)\right)$, where $\mu$ is the continuous, strictly increasing, and concave function measuring the planner's aversion to intrinsic risk. Thus, when $\varepsilon \rightarrow 0$, the consumption at the catastrophic state of nature vanishes, i.e. $x_{B}^{*}=\varepsilon\left(1-x_{0}^{*}\right) \rightarrow 0$, while $x_{0}^{*}$ remains positive for any finite degree of concavity of $\mu$. Therefore, $\lim _{\varepsilon \rightarrow 0} \pi_{B}\left(\frac{\varepsilon\left(1-c_{0}^{*}\right)}{\varepsilon\left(1-x_{0}^{*}\right)}\right)^{\gamma-1}=\pi_{B}\left(\frac{1-c_{0}^{*}}{1-x_{0}^{*}}\right)^{\gamma-1}$ is finite and the expected benefit of transferring resources to the future, RHS of (7.1), is bounded (independently of how large $\pi_{B}$ is).

\section{Conclusions}

The assessment of risky intergenerational prospects is crucial for policy evaluations in many economic models and, in particular, when dealing with climate change. This paper addresses such evaluations by studying the welfare economics of intergenerational risk and proposes an alternative to commonly used criteria, such as (discounted) expected utilitarianism.

The fair intergenerational utilitarian criterion measures social welfare in terms of the ratio between each conditional generation's consumption and an endogenous reference, named fair prospect. This reference interprets an ideal of equity and efficiency: the fair prospect allows evaluating consumptions based on how costly it is to transfer resources to each generation at each possible history; importantly, the society is not required to distribute resources according to this egalitarian reference. While the representation result holds more generally, I advocate for an efficient reference prospect that satisfies interim egalitarianism, a new fairness condition that combines both ex-ante and ex-post concerns for distributional equity. Interim egalitarianism requires that the lottery of each generation should be considered fair at each decision 
node (based on the information available at the time of the decision), both before risk is resolved and after any partial risk is resolved.

The introduction of a reference for the evaluation allows disentangling two sources of risk that the ethical observer can, and arguably should, treat differently. Intrinsic risk is unavoidable and determined by the specific resource distribution problem: the magnitude of the technological shocks and the timing of their resolution constrain what consumptions can be assigned to each conditional generation; aversion to these inequalities across possible histories is accounted for by the choice of the fair prospect. Instead, option risk follows from the ethical observer's choice to achieve a higher social welfare by deviating from the egalitarian reference; aversion to option risk measures the social tolerance for redistributing risk across possible histories in terms of deviation from the fair prospect.

By evaluating each generation's lottery in terms of the suggested fair prospect, the ethical observer agrees that the consumption corresponding to histories with tighter resource constraints -as those following catastrophic events- should be lower than at resource-abundant histories. The worse the technology at a possible history is, the more costly it is to improve consumptions at that history and, consequently, the smaller is the legitimate claim to a large consumption. This relativistic evaluation of risky prospects has three further consequences: (i) the society prefers an early resolution of uncertainty as it makes future choices simpler in terms of equity; (ii) the discount structure is endogenous (i.e. exponential, quasi-hyperbolic, or hyperbolic) and depends on technological risk, its resolution over time, and on the planner's aversion to it; (iii) the oversensitivity of other criteria to fat-tailed catastrophic events is always avoided, independently of probabilities and for any finite degree of inequality and risk aversion.

\section{Appendix A. Welfare REPRESEnTAtion: CONTINUity AND SEPARABilities}

Let the subdomain of decision trees $\mathcal{D}^{3} \subset \mathcal{D}$ to be such that for each $D \in \mathcal{D}^{3}$, $|T| \geq 3$ and $\left|N_{1}\right| \geq 3$. This restriction guarantees that the number of periods and the number of nodes per period (apart from the initial one) is at least 3. On this

subdomain, the functional form $V(c ; D)=\sum_{t \in T} q_{t}\left(\sum_{n \in N_{t}} u_{n}\left(c_{n}\right)\right)$ with continuous functions $q_{t}$ and $u_{n}$ representing each $\succsim_{D}$ can be obtained from 3 independent requirements, which I introduce next. 
The first one is continuity of the welfare evaluation, i.e. small variations of the consumption prospect should be associated with small variations of social welfare.

Continuity. For each $D \in \mathcal{D}$ and each $c \in C(D)$, the sets $\left\{\bar{c} \in C(D) \mid \bar{c} \succsim_{D} c\right\}$ and $\left\{\bar{c} \in C(D) \mid c \succsim_{D} \bar{c}\right\}$ are closed.

The second requirement is a separability condition across time. The ranking of two alternatives is independent of the consumption lottery assigned to a generation, if this is the same in both alternatives: unconcerned generations do not matter for the ranking.

Independence of unconcerned generations. For each $D \in \mathcal{D}$, each $\tilde{T} \subseteq T$, and each $c, c^{\prime}, c^{\prime \prime}, c^{\prime \prime \prime} \in C(D)$ such that:

(i) $c_{n}=c_{n}^{\prime}$ and $c_{n}^{\prime \prime}=c_{n}^{\prime \prime \prime}$ for each $t \in \tilde{T}$ and each $n \in N_{t}$;

(ii) $c_{n}=c_{n}^{\prime \prime}$ and $c_{n}^{\prime}=c_{n}^{\prime \prime \prime}$ for each $t \in T \backslash \tilde{T}$ and each $n \in N_{t}$;

then $c \succsim_{D} c^{\prime \prime}$ if and only if $c^{\prime} \succsim_{D} c^{\prime \prime \prime}$.

Assume that two prospects differ only with respect to the lottery assigned to generation $t \in T$. Then, the ranking of these alternatives is independent of the consumption assigned to a node, if this is the same in both alternatives: the consumption in unconcerned histories does not matter for the ranking.

Generation independence of unconcerned histories. For each $D \in \mathcal{D}$, each $t \in T$, each $\tilde{N}_{t} \subseteq N_{t}$, and each $c, c^{\prime}, c^{\prime \prime}, c^{\prime \prime \prime} \in C(D)$ such that:

(i) $c_{n}=c_{n}^{\prime}$ and $c_{n}^{\prime \prime}=c_{n}^{\prime \prime \prime}$ for each $n \in \tilde{N}_{t}$;

(ii) $c_{n}=c_{n}^{\prime \prime}$ and $c_{n}^{\prime}=c_{n}^{\prime \prime \prime}$ for each $n \in N \backslash \tilde{N}_{t}$;

then $c \succsim_{D} c^{\prime \prime}$ if and only if $c^{\prime} \succsim_{D} c^{\prime \prime \prime}$.

On the domain $D^{3}$, continuity and the above separability conditions imply the functional form assumed for social welfare, as the next result shows:

Lemma 1. On the domain $\mathcal{D}^{3}$, the social ordering function $\succsim$ satisfies continuity, independence of unconcerned generations, and generation independence of unconcerned histories if and only if for each $D \in \mathcal{D}^{3}$, there exist continuous functions $q_{t}$ (one for each $t \in T$ ) and $u_{n}$ (one for each $n \in N$ ) such that $\succsim_{D}$ is ordinally equivalent to $V(c ; D)=\sum_{t \in T} q_{t}\left(\sum_{n \in N_{t}} u_{n}\left(c_{n}\right)\right)$.

Proof. The result immediately obtains by applying Gorman (1968)'s separability theorem. The argument requires 3 periods, for the independence axiom over generations, and 3 nodes (excluding the first one) the independence condition of each generation across histories: these restrictions are satisfied on $\mathcal{D}^{3}$. 


\section{Appendix B. Proof of Theorem 1}

\section{$S 1$ implies $S 2$}

The proof is divided in 7 steps.

Assume $S 1$ and let $D \in \mathcal{D}$ and $x=\psi(D)$. The first step shows that the social ordering for $D, \succsim_{D}$, can be restricted to a functional representation with strictly increasing functions.

Step 1. For each $t \in T$ and each $n \in N_{t}$, there exist continuous and strictly increasing functions $q_{t}$ (one for each $t \in T$ ) and $u_{n}$ (one for each $n$ ) such that $\succsim_{D}$ is ordinally equivalent to:

$$
V(c ; D)=\sum_{t \in T} q_{t}\left(\sum_{n \in N_{t}} u_{n}\left(c_{n}\right)\right)
$$

Proof. By the restriction of the welfare representation of each $\succsim_{D}$, functions $q_{t}$ (one for each $t \in T$ ) and $u_{n}$ (one for each $n$ ) are strictly monotonic and continuous. By strict monotonicity, it must be true that either $q_{t}$ and $u_{n}$ are all strictly increasing for each $t \in T$ and each $n \in N_{t}$ or these are all strictly decreasing. Either choices lead to ordinally equivalent social orderings; following the usual convention, let all functions be strictly increasing.

The next step shows that, when combining risk-balancing and proportionality with the representation of Step 1, the utility function of each conditional generation $\left(u_{n}\right)$ can be rewritten in terms of the "relative consumption" $c_{n} / x_{n}$. Moreover, such utility is strictly concave in $c_{n} / x_{n}$ and is equal across nodes at the same period up to an additive constant.

Step 2. For each $t \in T$, there exist strictly increasing and strictly concave function $w_{t}: \mathbb{R}_{+} \rightarrow \mathbb{R}_{+}$such that:

$$
w_{t}(\alpha)=\frac{u_{n}\left(\alpha x_{n}\right)}{\pi_{n} x_{n}}+\chi_{n} \text { for some } \chi_{n} \in \mathbb{R} \text {, each } \alpha \in \mathbb{R}_{+} \text {, and each } n \in N_{t} \text {. }
$$

Proof. For each $t \in T$, each $n \in N_{t}$, and each $c_{n} \in \mathbb{R}_{+}$define $w_{n}\left(\frac{c_{n}}{x_{n}}\right) \equiv \frac{u_{n}\left(c_{n}\right)}{\pi_{n} x_{n}}$; note that for each $n \in N, x_{n}>0$ as shown in Corollary 1. By Step 1, $u_{n}$ is strictly increasing; thus, $w_{n}$ is strictly increasing and almost everywhere differentiable. Let a pair $c, \bar{c} \in C(D)$ be such that for some $t \in T$, a pair $n, n^{\prime} \in N_{t}$ and a $\delta \in \mathbb{R}_{+}$ the following conditions hold: (i) $c_{n}=\bar{c}_{n}-\frac{\delta}{\pi_{n}} \geq x_{n}$; (ii) $c_{n^{\prime}}=\bar{c}_{n^{\prime}}+\frac{\delta}{\pi_{n^{\prime}}} \leq x_{n^{\prime}}$; (iii) 
$c_{n^{\prime \prime}}=\bar{c}_{n^{\prime \prime}}$ for each $n^{\prime \prime} \neq n, n^{\prime}$. By risk-balancing, $c \succ_{D} \bar{c}$. By Step 1, this requires that $V(c ; D)-V(\bar{c} ; D)>0$ or, using (iii), that:

$$
\left[u_{n}\left(c_{n}\right)-u_{n}\left(c_{n}-\frac{\delta}{\pi_{n}}\right)\right]+\left[u_{n^{\prime}}\left(c_{n^{\prime}}\right)-u_{n^{\prime}}\left(c_{n^{\prime}}+\frac{\delta}{\pi_{n^{\prime}}}\right)\right]>0
$$

Substituting the utility functions $w_{n}$ and $w_{n^{\prime}}$ in (B.2), gives:

$$
\begin{gathered}
\pi_{n} x_{n}\left[w_{n}\left(\frac{c_{n}}{x_{n}}\right)-w_{n}\left(\frac{c_{n}}{x_{n}}-\frac{\delta}{\pi_{n} x_{n}}\right)\right]+ \\
+\pi_{n^{\prime}} x_{n^{\prime}}\left[w_{n^{\prime}}\left(\frac{c_{n^{\prime}}}{x_{n^{\prime}}}\right)-w_{n^{\prime}}\left(\frac{c_{n^{\prime}}}{x_{n^{\prime}}}+\frac{\delta}{\pi_{n^{\prime}} x_{n^{\prime}}}\right)\right]>0
\end{gathered}
$$

If $w_{n}$ and $w_{n^{\prime}}$ are differentiable at $\frac{c_{n}}{x_{n}}$ and $\frac{c_{n^{\prime}}}{x_{n^{\prime}}}$, dividing by $\delta$ and taking the limit for $\delta \rightarrow 0$, yields:

$$
w_{n}^{\prime}\left(\frac{c_{n}}{x_{n}}\right)<w_{n^{\prime}}^{\prime}\left(\frac{c_{n^{\prime}}}{x_{n^{\prime}}}\right) .
$$

Since $w_{n}, w_{n^{\prime}}$ are differentiable almost everywhere, condition (B.3) holds almost everywhere for each $\frac{c_{n}}{x_{n}}>1>\frac{c_{n^{\prime}}}{x_{n^{\prime}}}$ and, symmetrically, the reverse inequality holds almost everywhere for each $\frac{c_{n}}{x_{n}}<1<\frac{c_{n^{\prime}}}{x_{n^{\prime}}}$. Thus, if the functions are differentiable at $1, w_{n}^{\prime}(1)=w_{n^{\prime}}^{\prime}(1)$.

Let $\alpha>0$ and $D^{\alpha} \in \mathcal{D}$ be such that $\psi\left(D^{\alpha}\right)=\alpha \psi(D)$. By the previous steps and proportionality, $V(c ; D)>V(\bar{c} ; D)$ if and only if $V\left(c ; D^{\alpha}\right)>V\left(\bar{c} ; D^{\alpha}\right)$. Since this equivalence holds for each $\alpha>0$, equation (B.3) holds almost everywhere for each $\frac{c_{n}}{x_{n}}>\alpha>\frac{c_{n^{\prime}}}{x_{n^{\prime}}}$ and each $\alpha>0$. Thus $w_{n}^{\prime}(\alpha)=w_{n^{\prime}}^{\prime}(\alpha)$ almost everywhere for each $\alpha>0$ and $w_{n}$ and $w_{n^{\prime}}$ are strictly concave. This also implies that for each $t \in T$, there exists a strictly increasing and strictly concave function $w_{t}: \mathbb{R}_{+} \rightarrow \mathbb{R}_{+}$such that for each $n \in N_{t}$ there exists a constant $\chi_{n} \in \mathbb{R}$ for which $w_{t}(\alpha)=w_{n}(\alpha)+\chi_{n}$ for each $\alpha \in \mathbb{R}_{+}$.

The next step combines intergenerational equity and proportionality with the representation of Step 1 to show the effect of marginally increasing a relative consumption equally assigned to all conditional generations at different periods. For notational convenience, let $\chi_{t} \equiv \sum_{n \in N_{t}} \chi_{n}$.

Step 3. For each $t \in T$ and almost each $\alpha \in \mathbb{R}_{+}$,

$$
q_{t}^{\prime}\left(\chi_{t}+\sum_{n \in N_{t}} \pi_{n} x_{n} w_{t}(\alpha)\right) w_{t}^{\prime}(\alpha) \beta^{-t}=q_{t^{\prime}}^{\prime}\left(\chi_{t^{\prime}}+\sum_{n^{\prime} \in N_{t^{\prime}}} \pi_{n^{\prime}} x_{n^{\prime}} w_{t^{\prime}}(\alpha)\right) w_{t^{\prime}}^{\prime}(\alpha) \beta^{-t^{\prime}}
$$


Proof. Let a pair $c, \bar{c} \in C(D)$ be such that for some $t, t^{\prime} \in T$ and $a, b, \delta \in \mathbb{R}_{+}$: (i) $\frac{c_{n}}{x_{n}}=\frac{\bar{c}_{n}}{x_{n}}-\frac{\delta}{\beta^{t} x_{n}}=a \geq 1$ for each $n \in N_{t}$; (ii) $\frac{c_{n^{\prime}}}{x_{n^{\prime}}}=\frac{\bar{c}_{n^{\prime}}}{x_{n^{\prime}}}+\frac{\delta}{\beta^{t^{\prime}} x_{n^{\prime}}}=b \leq 1$ for each $n^{\prime} \in N_{t^{\prime}}$; (iii) $c_{n^{\prime \prime}}=\bar{c}_{n^{\prime \prime}}$ for each $n^{\prime \prime} \in N \backslash\left\{N_{t} \bigcup N_{t^{\prime}}\right\}$. Since $c$ is obtained by $\bar{c}$ via a progressive intergenerational transfer, intergenerational equity implies that $c \succsim_{D} \bar{c}$. By Step 1 , this requires that $V(c ; D)-V(\bar{c} ; D) \geq 0$ or, using (iii), that:

$$
\begin{aligned}
& q_{t}\left(\sum_{n \in N_{t}} u_{n}\left(c_{n}\right)\right)+q_{t^{\prime}}\left(\sum_{n^{\prime} \in N_{t^{\prime}}} u_{n^{\prime}}\left(c_{n^{\prime}}\right)\right) \geq \\
& q_{t}\left(\sum_{n \in N_{t}} u_{n}\left(c_{n}-\frac{\delta}{\beta^{t}}\right)\right)+q_{t^{\prime}}\left(\sum_{n^{\prime} \in N_{t^{\prime}}} u_{n^{\prime}}\left(c_{n^{\prime}}+\frac{\delta}{\beta^{t^{\prime}}}\right)\right) .
\end{aligned}
$$

Substituting the functions $w_{t}, w_{t^{\prime}}$ (see Step 2) in (B.4) yields:

$$
\begin{aligned}
& q_{t}\left(\chi_{t}+\sum_{n \in N_{t}} \pi_{n} x_{n} w_{t}(a)\right)-q_{t}\left(\chi_{t}+\sum_{n \in N_{t}} \pi_{n} x_{n} w_{t}\left(a-\frac{\delta}{\beta^{t} x_{n}}\right)\right)+ \\
& q_{t^{\prime}}\left(\chi_{t^{\prime}}+\sum_{n^{\prime} \in N_{t^{\prime}}} \pi_{n^{\prime}} x_{n^{\prime}} w_{t^{\prime}}(b)\right)-q_{t^{\prime}}\left(\chi_{t^{\prime}}+\sum_{n^{\prime} \in N_{t^{\prime}}} \pi_{n^{\prime}} x_{n^{\prime}} w_{t^{\prime}}\left(b+\frac{\delta}{\beta^{t^{\prime}} x_{n^{\prime}}}\right)\right) \geq 0
\end{aligned}
$$

Assume $q_{t}$ is differentiable at $z_{t}(a) \equiv \chi_{t}+\sum_{n \in N_{t}} \pi_{n} x_{n} w_{t}(a)$ and $q_{t^{\prime}}$ is differentiable at $z_{t^{\prime}}(b) \equiv \chi_{t^{\prime}}+\sum_{n^{\prime} \in N_{t^{\prime}}} \pi_{n^{\prime}} x_{n^{\prime}} w_{t^{\prime}}(b)$; assume $w_{t}$ and $w_{t^{\prime}}$ are differentiable at $a$ and $b$ respectively. Then, dividing the above by $\delta$ and taking the limit for $\delta \rightarrow 0$, implies that for each $a \geq 1 \geq b, q_{t}^{\prime}\left(z_{t}\right) w_{t}^{\prime}(a) \beta^{-t} \leq q_{t^{\prime}}^{\prime}\left(z_{t^{\prime}}\right) w_{t^{\prime}}^{\prime}(b) \beta^{-t^{\prime}}$. This holds almost everywhere for each $a \geq 1 \geq b$ (and, symmetrically, the reverse inequality holds whenever $a \leq 1 \leq b)$. By proportionality (similar to Step 2), $q_{t}^{\prime}\left(z_{t}(\alpha)\right) w_{t}^{\prime}(\alpha) \beta^{-t}=$ $q_{t^{\prime}}^{\prime}\left(z_{t^{\prime}}(\alpha)\right) w_{t^{\prime}}^{\prime}(\alpha) \beta^{-t^{\prime}}$ for almost all $\alpha \in \mathbb{R}_{+}$. Using the definitions of $z_{t}(\alpha)$ and $z_{t^{\prime}}(\alpha)$, the statement of Step 3 follows.

The next step shows differentiability of the evaluation functions.

Step 4. For each $t \in T, w_{t}$ and $q_{t}$ are differentiable.

Proof. Let $t \in T$. By contradiction, assume $w_{t}$ is not differentiable at $\bar{\alpha} \in \mathbb{R}_{+}$: then left and right derivative at $\bar{\alpha}$ are such that $w_{t}^{\prime}\left(\bar{\alpha}^{-}\right) \neq w_{t}^{\prime}\left(\bar{\alpha}^{+}\right)$. By continuity and almost everywhere differentiability of $w_{t}$, there exist a pair $c, \bar{c} \in C(D)$ such that: (i) $c_{n}>\bar{c}_{n}=\bar{\alpha}=\bar{c}_{n^{\prime}}>c_{n^{\prime}}$ for some $n, n^{\prime} \in N_{t}$; (ii) $c_{n^{\prime \prime}}=\bar{c}_{n^{\prime \prime}}$ for each $n^{\prime \prime} \neq n, n^{\prime}$; (iii) $V(c ; D)=V(\bar{c} ; D)$; and (iv) $w_{t}$ is differentiable at $c_{n}$ and $c_{n^{\prime}}$. Define $\Delta V \equiv V(c ; D)-V(\bar{c} ; D)$; by the previous steps and $(i i i)$ :

$$
\Delta V=\left[\pi_{n} x_{n} w_{t}\left(\frac{c_{n}}{x_{n}}\right)+\pi_{n^{\prime}} x_{n^{\prime}} w_{t}\left(\frac{c_{n^{\prime}}}{x_{n^{\prime}}}\right)\right]-\left[\pi_{n} x_{n} w_{t}(\bar{\alpha})+\pi_{n^{\prime}} x_{n^{\prime}} w_{t}(\bar{\alpha})\right]=0 .
$$

Let $\alpha>0$ and $D^{\alpha} \in \mathcal{D}$ be such that $\phi(D)=\alpha \phi\left(D^{\alpha}\right)$. For each $\alpha>0$, let $\Delta V^{\alpha} \equiv$ $V\left(c ; D^{\alpha}\right)-V\left(\bar{c} ; D^{\alpha}\right)$ or, substituting: 


$$
\begin{gathered}
\Delta V^{\alpha}=\alpha\left[\pi_{n} x_{n} w_{t}\left(\frac{c_{n}}{\alpha x_{n}}\right)+\pi_{n^{\prime}} x_{n^{\prime}} w_{t}\left(\frac{c_{n^{\prime}}}{\alpha x_{n^{\prime}}}\right)\right]+ \\
-\alpha\left[\pi_{n} x_{n} w_{t}\left(\frac{\bar{\alpha}}{\alpha}\right)+\pi_{n^{\prime}} x_{n^{\prime}} w_{t}\left(\frac{\bar{\alpha}}{\alpha}\right)\right]=0 .
\end{gathered}
$$

By differentiability of $w_{t}$ at $\frac{c_{n}}{x_{n}}$ and $\frac{c_{n^{\prime}}}{x_{n^{\prime}}}$ and by $w_{t}^{\prime}\left(\bar{\alpha}^{-}\right) \neq w_{t}^{\prime}\left(\bar{\alpha}^{+}\right)$, it follows that $\lim _{\alpha \rightarrow 1^{-}} \Delta V^{\alpha} \neq \lim _{\alpha \rightarrow 1^{+}} \Delta V^{\alpha}$. Thus proportionality cannot hold, i.e. there exists $\alpha>0$ such that $\Delta V \neq \Delta V^{\alpha}$. This contradiction proves differentiability of $w_{t}$.

Similarly, assume $q_{t}$ is not differentiable at $z$, so that $q_{t}^{\prime}\left(z^{-}\right) \neq q_{t}^{\prime}\left(z^{+}\right)$. Since $w_{t}$ is continuous and strictly increasing (and thus invertible), there exists a $\bar{\alpha} \in \mathbb{R}_{+}$such that $z=\chi_{t}+\sum_{n \in N_{t}} \pi_{n} x_{n} w_{t}(\bar{\alpha})$. Let $t^{\prime} \neq t$. By the result of Step 3, also $q_{t^{\prime}}$ is not differentiable at $\chi_{t^{\prime}}+\sum_{n^{\prime} \in N_{t^{\prime}}} \pi_{n^{\prime}} x_{n^{\prime}} w_{t^{\prime}}(\bar{\alpha})$. By continuity and almost everywhere differentiability of $q_{t}, q_{t^{\prime}}, w_{t}, w_{t^{\prime}}$, there exists a pair $c, \bar{c} \in C(D)$ and a pair $a, b \in$ $\mathbb{R}_{+}$such that: (i) $c_{n}=a>\bar{c}_{n}=\bar{\alpha}=\bar{c}_{n^{\prime}}>b=c_{n^{\prime}}$ for each $n \in N_{t}$ and each $n^{\prime} \in N_{t^{\prime}}$; (ii) $c_{n^{\prime \prime}}=\bar{c}_{n^{\prime \prime}}$ for each $n^{\prime \prime} \in N \backslash\left\{N_{t} \bigcup N_{t^{\prime}}\right\}$; (iii) $V(c ; D)=V(\bar{c} ; D)$; and (iv) $w_{t}$ is differentiable at $a, q_{t}$ is differentiable at $y_{t} \equiv \chi_{t}+\sum_{n \in N_{t}} \pi_{n} x_{n} w_{t}(a), w_{t^{\prime}}$ is differentiable at $b$, and $q_{t^{\prime}}$ is differentiable at $y_{t^{\prime}} \equiv \chi_{t^{\prime}}+\sum_{n^{\prime} \in N_{t^{\prime}}} \pi_{n^{\prime}} x_{n^{\prime}} w_{t^{\prime}}(b)$. As above, this leads to $\lim _{\alpha \rightarrow 1^{-}} \Delta V^{\alpha} \neq \lim _{\alpha \rightarrow 1^{+}} \Delta V^{\alpha}$; thus, there exists an $\alpha>0$ such that proportionality is contradicted, proving the differentiability of $q_{t}$.

The next results tells that, for each $t \in T$, the functions $w_{t}$ have a power functional form (with the logs being a special case with power 0 ).

Step 5. There exist constants $\bar{\eta}_{t}, \eta_{t}, \tilde{\gamma}_{t} \in \mathbb{R}$ (a triplet for each $t \in T$ ) such that for each $t \in T$ :

$$
w_{t}\left(\frac{c_{n}}{x_{n}}\right)= \begin{cases}\bar{\eta}_{t}+\frac{\eta_{t}}{1-\tilde{\gamma}_{t}}\left(\frac{c_{n}}{x_{n}}\right)^{1-\tilde{\gamma}_{t}} & \text { if } \tilde{\gamma}_{t} \neq 1 \\ \bar{\eta}_{t}+\eta_{t} \ln \left(\frac{c_{n}}{x_{n}}\right) & \text { if } \tilde{\gamma}_{t}=1\end{cases}
$$

Proof. Let a pair $c, \bar{c} \in C(D)$ be such that: (i) $c \sim_{D} \bar{c}$; (ii) $\sum_{n \in N_{t}} \pi_{n} x_{n} w_{t^{\prime}}\left(\frac{c_{n}}{x_{n}}\right)=$ $\sum_{n \in N_{t}} \pi_{n} x_{n} w_{t^{\prime}}\left(\frac{\bar{c}_{n}}{x_{n}}\right)$ for each $t \in T$; and (iii) $c_{n}=\bar{c}_{n}$ for each $n \neq n^{\prime}, n^{\prime \prime}$, with $n^{\prime}, n^{\prime \prime} \in N_{t}$ for some $t \in T$. This yields:

$$
\pi_{n^{\prime}} x_{n^{\prime}} w_{t}\left(\frac{c_{n^{\prime}}}{x_{n^{\prime}}}\right)+\pi_{n^{\prime \prime}} x_{n^{\prime \prime}} w_{t}\left(\frac{c_{n^{\prime \prime}}}{x_{n^{\prime \prime}}}\right)=\pi_{n^{\prime}} x_{n^{\prime}} w_{t}\left(\frac{\bar{c}_{n^{\prime}}}{x_{n^{\prime}}}\right)+\pi_{n^{\prime \prime}} x_{n^{\prime \prime}} w_{t}\left(\frac{\bar{c}_{n^{\prime \prime}}}{x_{n^{\prime \prime}}}\right)
$$

and, by proportionality, also:

$$
\pi_{n^{\prime}} x_{n^{\prime}} w_{t}\left(\frac{c_{n^{\prime}}}{\alpha x_{n^{\prime}}}\right)+\pi_{n^{\prime \prime}} x_{n^{\prime \prime}} w_{t}\left(\frac{c_{n^{\prime \prime}}}{\alpha x_{n^{\prime \prime}}}\right)=\pi_{n^{\prime}} x_{n^{\prime}} w_{t}\left(\frac{\bar{c}_{n^{\prime}}}{\alpha x_{n^{\prime}}}\right)+\pi_{n^{\prime \prime}} x_{n^{\prime \prime}} w_{t}\left(\frac{\bar{c}_{n^{\prime \prime}}}{\alpha x_{n^{\prime \prime}}}\right)
$$


Let $\delta^{\prime} \equiv \bar{c}_{n^{\prime}}-c_{n^{\prime}}, \delta^{\prime \prime} \equiv \bar{c}_{n^{\prime \prime}}-c_{n^{\prime \prime}}$. By implicit differentiation, equation (B.5) holds at the limit for $\delta^{\prime} \rightarrow 0$, when $\frac{\partial \delta^{\prime \prime}}{\partial \delta^{\prime}}=-\frac{\pi_{n^{\prime}} w_{t}^{\prime}\left(\frac{c_{n^{\prime}}}{x_{n^{\prime}}}\right)}{\pi_{n^{\prime \prime}} w_{t}^{\prime}\left(\frac{c_{n} n^{\prime \prime}}{x_{n^{\prime \prime}}}\right)}$. Divide (B.6) by $\delta^{\prime}$, let $\partial \delta^{\prime \prime}=$ $-\frac{\pi_{n^{\prime}} w_{t}^{\prime}\left(\frac{c_{n^{\prime}}}{x_{n^{\prime}}}\right)}{\pi_{n^{\prime \prime}} w_{t}^{\prime}\left(\frac{c_{n^{\prime \prime}}}{x_{n^{\prime \prime}}}\right)} \partial \delta^{\prime}$, and take the limit for $\delta^{\prime} \rightarrow 0$. Then:

$$
\frac{w_{t}^{\prime}\left(\frac{c_{n^{\prime}}}{\alpha x_{n^{\prime}}}\right)}{w_{t}^{\prime}\left(\frac{c_{n^{\prime}}}{x_{n^{\prime}}}\right)}=\frac{w_{t}^{\prime}\left(\frac{c_{n^{\prime \prime}}}{\alpha x_{n^{\prime \prime}}}\right)}{w_{t}^{\prime}\left(\frac{c_{n^{\prime \prime}}}{x_{n^{\prime \prime}}}\right)}
$$

Define the continuous function $\lambda_{t}(\alpha)=\frac{w_{t}^{\prime}\left(\frac{c_{n^{\prime \prime}}}{\alpha x_{n^{\prime \prime}}}\right)}{w_{t}^{\prime}\left(\frac{c_{n} n^{\prime \prime}}{x_{n^{\prime \prime}}}\right)}$ (since $w_{t}^{\prime}>0$, this is positive). Substituting in (B.7) and taking the log transformation gives:

$$
\ln w_{t}^{\prime}\left(\frac{c_{n^{\prime}}}{\alpha x_{n^{\prime}}}\right)-\ln w_{t}^{\prime}\left(\frac{c_{n^{\prime}}}{x_{n^{\prime}}}\right)=\ln \lambda_{t}(\alpha) .
$$

Let $g(\ln (z)) \equiv w_{t}^{\prime}(z)$ for each $z>0$. Substitute, divide by $\ln \alpha$ and take the limit for $\ln \alpha \rightarrow 0$ to obtain:

$$
\frac{d \ln g\left(\ln \frac{c_{n^{\prime}}}{x_{n^{\prime}}}\right)}{d \ln \frac{c_{n^{\prime}}}{x_{n^{\prime}}}}=-\lim _{\ln \alpha \rightarrow 0} \frac{\ln \lambda_{t}(\alpha)}{\ln \alpha} .
$$

The RHS is finite by differentiability of $w_{t}$. Let $\tilde{\gamma}_{t} \equiv \lim _{\ln \alpha \rightarrow 0} \frac{\ln \lambda_{t}(\alpha)}{\ln \alpha}$ and let $\eta_{t}$ be the integrating constant. Integrating w.r.t. $\frac{c_{n^{\prime}}}{x_{n^{\prime}}}$ gives:

$$
g\left(\ln \frac{c_{n^{\prime}}}{x_{n^{\prime}}}\right)=w_{t}^{\prime}\left(\frac{c_{n^{\prime}}}{x_{n^{\prime}}}\right)=\eta_{t}\left(\frac{c_{n^{\prime}}}{x_{n^{\prime}}}\right)^{-\tilde{\gamma}_{t}}
$$

Further integrating - let $\bar{\eta}_{t}$ be the additive constant - implies that $w_{t}\left(\frac{c_{n}}{x_{n}}\right)=\bar{\eta}_{t}+$ $\frac{\eta_{t}}{1-\tilde{\gamma}_{t}}\left(\frac{c_{n}}{x_{n}}\right)^{1-\tilde{\gamma}_{t}}$ if $\tilde{\gamma}_{t} \neq 1$ and $w_{t}\left(\frac{c_{n}}{x_{n}}\right)=\bar{\eta}_{t}+\eta_{t} \ln \left(\frac{c_{n}}{x_{n}}\right)$ otherwise.

For each $c \in C(D)$ and each $t \in T$, let

$$
\sigma_{t} \equiv\left[\left(1-\frac{\tilde{\gamma}_{t}}{1-\tilde{\gamma}_{t}}\right) \bar{\eta}_{t} \sum_{n \in N_{t}} \pi_{n} x_{n}+\frac{\tilde{\gamma}_{t}}{1-\tilde{\gamma}_{t}}\left(\sum_{n \in N_{t}} \pi_{n} x_{n} w_{t}\left(\frac{c_{n}}{x_{n}}\right)\right)\right] .
$$

The next step shows that also $q_{t}$ has a power functional form.

Step 6. There exist $a \tilde{\rho} \in \mathbb{R}$ and $\xi_{t} \in \mathbb{R}$ (one for eacht $\in T$ ) such that for each $t \in T$ : 


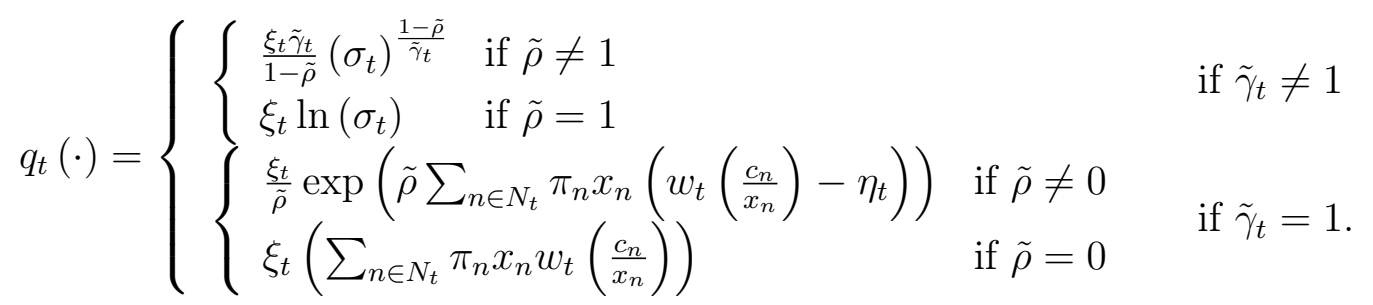

Proof. By proportionality, for each $c, \bar{c} \in C(D), c \succsim_{D} \bar{c}$ iff $c \succsim_{D^{\alpha}} \bar{c}$ and, by the previous steps, $V(c ; D) \geq V(\bar{c} ; D)$ iff $V\left(c ; D^{\alpha}\right) \geq V\left(\bar{c} ; D^{\alpha}\right)$. Then, there exists a function $\lambda: \mathbb{R}_{+} \rightarrow \mathbb{R}_{+}$, with $\lambda(1)=1$ and $\lambda(\alpha) \neq 0$, such that for each $\alpha>0$ :

$$
\begin{gathered}
\sum_{t \in T}\left[q_{t}\left(\chi_{t}+\sum_{n \in N_{t}} \pi_{n} x_{n} w_{t}\left(\frac{c_{n}}{x_{n}}\right)\right)-q_{t}\left(\chi_{t}+\sum_{n \in N_{t}} \pi_{n} x_{n} w_{t}\left(\frac{\bar{c}_{n}}{x_{n}}\right)\right)\right]= \\
=\lambda(\alpha) \sum_{t \in T}\left[q_{t}\left(\chi_{t}+\sum_{n \in N_{t}} \pi_{n} \alpha x_{n} w_{t}\left(\frac{c_{n}}{\alpha x_{n}}\right)\right)-q_{t}\left(\chi_{t}+\sum_{n \in N_{t}} \pi_{n} \alpha x_{n} w_{t}\left(\frac{\bar{c}_{n}}{\alpha x_{n}}\right)\right)\right]
\end{gathered}
$$

Assume that $c, \bar{c} \in C(D)$ are such that for some $t \in T$ and $\delta>0$ : (i) $\frac{c_{n}}{x_{n}}+\delta=\frac{\bar{c}_{n}}{x_{n}}$ for each $n \in N_{t}$; and (ii) $c_{n}=\bar{c}_{n}$ for each $n \in N \backslash N_{t}$. Define $\tilde{\pi}_{t} \equiv \sum_{n \in N_{t}} \pi_{n} x_{n}$. Equation (B.9), divided by $\delta$, simplifies as follows:

$$
\begin{gathered}
\frac{q_{t}\left(\chi_{t}+w_{t}\left(\frac{c_{n}}{x_{n}}+\delta\right) \tilde{\pi}_{t}\right)-q_{t}\left(\chi_{t}+w_{t}\left(\frac{c_{n}}{x_{n}}\right) \tilde{\pi}_{t}\right)}{\delta}= \\
=\lambda(\alpha) \frac{\left[q_{t}\left(\chi_{t}+\alpha w_{t}\left(\frac{c_{n}}{\alpha x_{n}}+\frac{\delta}{\alpha}\right) \tilde{\pi}_{t}\right)-q_{t}\left(\chi_{t}+\alpha w_{t}\left(\frac{c_{n}}{\alpha x_{n}}\right) \tilde{\pi}_{t}\right)\right]}{\delta} .
\end{gathered}
$$

At the limit for $\delta \rightarrow 0$, it gives:

$$
w_{t}^{\prime}\left(\frac{c_{n}}{x_{n}}\right) q_{t}^{\prime}\left(\chi_{t}+w_{t}\left(\frac{c_{n}}{x_{n}}\right) \tilde{\pi}_{t}\right)=\lambda(\alpha) w_{t}^{\prime}\left(\frac{c_{n}}{\alpha x_{n}}\right) q_{t}^{\prime}\left(\chi_{t}+\alpha w_{t}\left(\frac{c_{n}}{\alpha x_{n}}\right) \tilde{\pi}_{t}\right)
$$

Case 1. Assume $w_{t}\left(\frac{c_{n}}{x_{n}}\right)=\bar{\eta}_{t}+\frac{\eta_{t}}{1-\tilde{\gamma}_{t}}\left(\frac{c_{n}}{x_{n}}\right)^{1-\tilde{\gamma}_{t}}$ with $\tilde{\gamma}_{t} \neq 1$. Then, substituting $w_{t}^{\prime}$ in (B.10) gives $q_{t}^{\prime}\left(\chi_{t}+w_{t}\left(\frac{c_{n}}{x_{n}}\right) \tilde{\pi}_{t}\right)=\lambda(\alpha) \alpha^{\tilde{\gamma}_{t}} q_{t}^{\prime}\left(\chi_{t}+\alpha w_{t}\left(\frac{c_{n}}{\alpha x_{n}}\right) \tilde{\pi}_{t}\right)$. Define $\bar{g}\left(\ln \left(z-\chi_{t}\right)\right) \equiv q_{t}^{\prime}(z)$ for each $z>0$; substitute $\bar{g}$, take the log transformation, and divide by $\ln \alpha$ :

$$
\frac{\ln \bar{g}\left(\ln \alpha+\ln w_{t}\left(\frac{c_{n}}{\alpha x_{n}}\right)+\ln \tilde{\pi}_{t}\right)-\ln \bar{g}\left(\ln w_{t}\left(\frac{c_{n}}{x_{n}}\right)+\ln \tilde{\pi}_{t}\right)}{\ln \alpha}=-\frac{\ln \lambda(\alpha)}{\ln \alpha}-\tilde{\gamma}_{t}
$$

By differentiability of $q_{t}$, the limit is finite: let $\tilde{\rho} \equiv 1+\lim _{\ln \alpha \rightarrow 0} \frac{\ln \lambda(\alpha)}{\ln \alpha}$. The limit writes: 


$$
\left(1-\frac{\eta_{t}\left(\frac{c_{n}}{x_{n}}\right)^{1-\tilde{\gamma}_{t}}}{\bar{\eta}_{t}+\frac{\eta_{t}}{1-\tilde{\gamma}_{t}}\left(\frac{c_{n}}{x_{n}}\right)^{1-\tilde{\gamma}_{t}}}\right) \frac{d \ln \bar{g}\left(\ln \left(z-\chi_{t}\right)\right)}{d\left(\ln \left(z-\chi_{t}\right)\right)}=1-\tilde{\rho}-\tilde{\gamma}_{t}
$$

and, rearranging and substituting back for $q_{t}^{\prime}(z)$, yields:

$$
d \ln q_{t}^{\prime}(z)=\left(1-\tilde{\rho}-\tilde{\gamma}_{t}\right)\left(\frac{z}{\left(1-\frac{\tilde{\gamma}_{t}}{1-\tilde{\gamma}_{t}}\right) \tilde{\pi}_{t} \bar{\eta}_{t}+\frac{\tilde{\gamma}_{t}}{1-\tilde{\gamma}_{t}} z}\right) d\left(\ln \left(z-\chi_{t}\right)\right)
$$

By strict concavity of $w_{t}$ (see Step 2), $\tilde{\gamma}_{t} \neq 0$. Integrating the above -let $\xi_{t}$ be the integrating constant- gives:

$$
q_{t}^{\prime}(z)=\xi_{t}\left[\left(1-\frac{\tilde{\gamma}_{t}}{1-\tilde{\gamma}_{t}}\right) \tilde{\pi}_{t} \bar{\eta}_{t}+\frac{\tilde{\gamma}_{t}}{1-\tilde{\gamma}_{t}}\left(z-\chi_{t}\right)\right]^{\left(\frac{1-\tilde{\rho}}{\tilde{\gamma}_{t}}-1\right)}
$$

Further integrating (the additive constant is left out as ordinally irrelevant for the ranking) implies that:

$$
\begin{gathered}
q_{t}(z)=\frac{\xi_{t} \tilde{\gamma}_{t}}{1-\tilde{\rho}}\left[\left(1-\frac{\tilde{\gamma}_{t}}{1-\tilde{\gamma}_{t}}\right) \tilde{\pi}_{t} \bar{\eta}_{t}+\frac{\tilde{\gamma}_{t}}{1-\tilde{\gamma}_{t}}\left(z-\chi_{t}\right)\right]^{\frac{1-\tilde{\gamma}}{\tilde{\gamma}}} \text { if } \tilde{\rho} \neq 1 \text { and } \\
q_{t}(z)=\xi_{t} \ln \left[\left(1-\frac{\tilde{\gamma}_{t}}{1-\tilde{\gamma}_{t}}\right) \tilde{\pi}_{t} \bar{\eta}_{t}+\frac{\tilde{\gamma}_{t}}{1-\tilde{\gamma}_{t}}\left(z-\chi_{t}\right)\right] \text { otherwise. }
\end{gathered}
$$

Substituting $z=\chi_{t}+w_{t}\left(\frac{c_{n}}{x_{n}}\right) \tilde{\pi}_{t}$, gives the specified functional forms.

Case 2. Assume $w_{t}\left(\frac{c_{n}}{x_{n}}\right)=\bar{\eta}_{t}+\eta_{t} \ln \left(\frac{c_{n}}{x_{n}}\right)$. Substituting $w_{t}$ and $w_{t}^{\prime}$ in (B.10) gives: $q_{t}^{\prime}\left(\tilde{\pi}_{t} w_{t}\left(\frac{c_{n}}{x_{n}}\right)\right)=\alpha \lambda(\alpha) q_{t}^{\prime}\left(\alpha \tilde{\pi}_{t} w_{t}\left(\frac{c_{n}}{\alpha x_{n}}\right)\right)$. As above, substitute $\bar{g}$, take the $\log$ transformation, and divide by $\ln \alpha$ to obtain:

$$
\frac{\ln \bar{g}\left(\ln \alpha+\ln \tilde{\pi}_{t}+\ln w_{t}\left(\frac{c_{n}}{\alpha x_{n}}\right)\right)-\ln \bar{g}\left(\ln \tilde{\pi}_{t}+\ln w_{t}\left(\frac{c_{n}}{x_{n}}\right)\right)}{\ln \alpha}=-\frac{\ln \lambda(\alpha)}{\ln \alpha}-1
$$

Taking the limit for $\ln \alpha \rightarrow 0$, gives:

$$
\frac{d \ln \bar{g}\left(\ln \left(z-\chi_{t}\right)\right)}{d\left(\ln \left(z-\chi_{t}\right)\right)}\left[1-\frac{\eta_{t}}{\bar{\eta}_{t}+\eta_{t} \ln \left(\frac{c_{n}}{x_{n}}\right)}\right]=\frac{d \ln \bar{g}\left(\ln \left(z-\chi_{t}\right)\right)}{d\left(\ln \left(z-\chi_{t}\right)\right)}\left[1-\frac{\tilde{\pi}_{t} \eta_{t}}{z}\right]=\tilde{\rho}
$$

Thus:

$$
\int d \ln q_{t}^{\prime}(z)=\tilde{\rho} \int\left(\frac{z-\chi_{t}}{z-\chi_{t}-\tilde{\pi}_{t} \eta_{t}}\right) d\left(\ln \left(z-\chi_{t}\right)\right)
$$


Integrating a first time -let $\xi_{t}$ be the integrating constant- gives:

$$
q_{t}^{\prime}(z)=\xi_{t} \exp \left(\tilde{\rho} \sum_{n \in N_{t}} \pi_{n} x_{n}\left[w_{t}\left(\frac{c_{n}}{x_{n}}\right)-\eta_{t}\right]\right) .
$$

Integrating again (the additive constant is left out as ordinally irrelevant for the ranking) gives:

$$
\begin{cases}q_{t}=\frac{\xi_{t}}{\tilde{\rho}} \exp \left(\tilde{\rho} \sum_{n \in N_{t}} \pi_{n} x_{n}\left[w_{t}\left(\frac{c_{n}}{x_{n}}\right)-\eta_{t}\right]\right) & \text { if } \tilde{\rho} \neq 0 \\ q_{t}=\xi_{t}\left(\sum_{n \in N_{t}} \pi_{n} x_{n} w_{t}\left(\frac{c_{n}}{x_{n}}\right)\right) & \text { otherwise. }\end{cases}
$$

The next and last step of the proof combines the parametric functional forms with the concavity restrictions.

Step 7. The social ordering $\succsim_{D}$ can be represented by the fair intergenerational utilitarian criterion (4.3).

Proof. Let $c \in C(D)$ be such that $\frac{c_{n}}{x_{n}}=z$ for each $n \in N$. For each $t \in T$, let $\tilde{\pi}_{t} \equiv \sum_{n \in N_{t}} \pi_{n} x_{n}$. By Step 3 and 4 ,

$$
q_{t}^{\prime}\left(\sum_{n \in N_{t}} \pi_{n} x_{n} w_{t}(z)\right) w_{t}^{\prime}(z) \beta^{-t}=q_{t^{\prime}}^{\prime}\left(\sum_{n^{\prime} \in N_{t^{\prime}}} \pi_{n^{\prime}} x_{n^{\prime}} w_{t^{\prime}}(z)\right) w_{t^{\prime}}^{\prime}(z) \beta^{-t^{\prime}}
$$

for each $z \in \mathbb{R}_{+}$and each $t, t^{\prime} \in T$.

Case 1. Assume $w_{t}, w_{t^{\prime}}$ have the form of Step 5 with $\tilde{\gamma}_{t}, \tilde{\gamma}_{t^{\prime}} \neq 1$ : thus, $w_{t}(z)=$ $\bar{\eta}_{t}+\frac{\eta_{t}}{1-\tilde{\gamma}_{t}}(z)^{1-\tilde{\gamma}_{t}}$ and $w_{t^{\prime}}(z)=\bar{\eta}_{t^{\prime}}+\frac{\eta_{t^{\prime}}}{1-\tilde{\gamma}_{t^{\prime}}}(z)^{1-\tilde{\gamma}_{t^{\prime}}}$. Then:

$$
\begin{aligned}
& \xi_{t} \beta^{-t} \eta_{t}\left(\left(1-\frac{\tilde{\gamma}_{t}}{1-\tilde{\gamma}_{t}}\right) \tilde{\pi}_{t} \bar{\eta}_{t}+\frac{\tilde{\gamma}_{t}}{\left(1-\tilde{\gamma}_{t}\right)^{2}} \tilde{\pi}_{t} \eta_{t} z^{1-\tilde{\gamma}_{t}}\right)^{\frac{1-\tilde{\rho}}{\tilde{\gamma}_{t}}-1}(z)^{-\tilde{\gamma}_{t}}= \\
= & \xi_{t^{\prime}} \beta^{-t^{\prime}} \eta_{t^{\prime}}\left(\left(1-\frac{\tilde{\gamma}_{t}}{1-\tilde{\gamma}_{t}}\right) \tilde{\pi}_{t^{\prime}} \bar{\eta}_{t}+\frac{\tilde{\gamma}_{t^{\prime}}}{\left(1-\tilde{\gamma}_{t^{\prime}}\right)^{2}} \tilde{\pi}_{t^{\prime}} \eta_{t^{\prime}} z^{1-\tilde{\gamma}_{t}}\right)^{\frac{1-\tilde{\rho}}{\tilde{\gamma}_{t^{\prime}}}-1}(z)^{-\tilde{\gamma}_{t^{\prime}}} .
\end{aligned}
$$

Since this holds for each $z>0,1-\tilde{\rho} \neq \tilde{\gamma}_{t}$ implies that $\bar{\eta}_{t}=0$ (and equivalently for $t^{\prime}$ ). When $1-\tilde{\rho}=\tilde{\gamma}_{t}, q_{t}$ is linear (and thus also $q_{t^{\prime}}$ for each $t^{\prime} \neq t$ ) and any choice of $\bar{\eta}_{t}$ is ordinally equivalent; thus we can again set $\bar{\eta}_{t}=0$. Equalizing the exponents of $z$ of the RHS and LHS, requires $\tilde{\gamma}_{t}=\tilde{\gamma}_{t^{\prime}} \equiv \tilde{\gamma}$. Thus, the above conditions simplifies as:

$$
\xi_{t} \beta^{-t} \eta_{t}\left(\frac{\tilde{\gamma}}{(1-\tilde{\gamma})^{2}} \tilde{\pi}_{t} \eta_{t}\right)^{\frac{1-\tilde{\rho}}{\tilde{\gamma}}-1}=\xi_{t^{\prime}} \beta^{-t^{\prime}} \eta_{t^{\prime}}\left(\frac{\tilde{\gamma}}{(1-\tilde{\gamma})^{2}} \tilde{\pi}_{t^{\prime}} \eta_{t^{\prime}}\right)^{\frac{1-\tilde{\rho}}{\tilde{\gamma}}-1}
$$

The equality holds when the parameters are as follows: $\eta_{t}=\frac{(1-\tilde{\gamma})^{2}}{\tilde{\gamma}} \tilde{\pi}_{t}^{-1}$ and $\xi_{t}=\frac{\beta^{t}}{\tilde{\pi}_{0}} \eta_{t}^{-1}$ (and similarly for $t^{\prime}$ ). Let $\gamma \equiv 1-\tilde{\gamma}, \rho \equiv 1-\frac{\gamma}{1-\gamma}(1-\tilde{\rho}), \tilde{\pi}_{0}=x_{0}$ and $\tilde{\pi}_{t}=\sum_{n \in N_{t}} \pi_{n} x_{n}$. 
Then, the FIU criterion arises, where for each $t \in T$ :

$$
v_{t}(c ; D) \equiv\left(\sum_{n \in N_{t}} \frac{\pi_{n} x_{n}}{\tilde{\pi}_{t}}\left(\frac{c_{n}}{x_{n}}\right)^{\gamma}\right)^{\frac{1}{\gamma}},
$$

and social welfare is:

$$
V(c ; D)=\left\{\begin{array}{cc}
\sum_{t \in T} \beta^{t} \frac{\tilde{\pi}_{t}}{\tilde{\pi}_{0}} \frac{\left(v_{t}(c ; D)\right)^{1-\rho}}{1-\rho} & \rho \geq 0, \rho \neq 1 \\
\sum_{t \in T} \beta^{t} \frac{\tilde{\pi}_{t}}{\tilde{\pi}_{0}} \ln \left(v_{t}(c ; D)\right) & \rho=1
\end{array}\right.
$$

Case 2. Assume $\tilde{\gamma}_{t}=1$ and $w_{t}=\bar{\eta}_{t}+\eta_{t} \ln \left(\frac{c_{n}}{x_{n}}\right)$ for some $t \in T$. By Case 1 , this also implies that $\tilde{\gamma}_{t^{\prime}}=1$ for each $t^{\prime} \in T$. Substitute in (B.11) the functional forms obtained from Step 5:

$$
\begin{aligned}
& \xi_{t} \beta^{-t} \eta_{t} \exp \left(\tilde{\rho} \sum_{n \in N_{t}} \pi_{n} x_{n}\left[\bar{\eta}_{t}+\eta_{t} \ln z-\eta_{t}\right]\right) z^{-1}= \\
= & \xi_{t^{\prime}} \beta^{-t^{\prime}} \eta_{t^{\prime}} \exp \left(\tilde{\rho} \sum_{n \in N_{t^{\prime}}} \pi_{n} x_{n}\left[\bar{\eta}_{t^{\prime}}+\eta_{t^{\prime}} \ln z-\eta_{t^{\prime}}\right]\right) z^{-1} .
\end{aligned}
$$

Since this holds for each $z>0, \tilde{\rho} \neq 0$ implies that $\bar{\eta}_{t}=\eta_{t}$ (and equivalently for $t^{\prime}$ ). When $\tilde{\rho}=0, q_{t}$ is linear and any choice of $\bar{\eta}_{t}$ is ordinally indifferent; thus set $\bar{\eta}_{t}=\eta_{t}$. Equality of RHS and LHS are ensured by $\eta_{t}=\tilde{\pi}_{t}^{-1}$ and $\xi_{t}=\frac{\beta^{t}}{\tilde{\pi}_{0}} \eta_{t}^{-1}$. Let $\rho \equiv 1-\tilde{\rho}$, $\tilde{\pi}_{0}=x_{0}, \tilde{\pi}_{t}=\sum_{n \in N_{t}} \pi_{n} x_{n}$, and substitute in the forms of Step 5 to get the FIU remaining cases, where for each $t \in T$ :

$$
v_{t}(c ; D) \equiv \prod_{n \in N_{t}}\left(\frac{c_{n}}{x_{n}}\right)^{\frac{\pi_{n} x_{n}}{\tilde{\pi}_{t}}}
$$

and:

$$
V(c ; D)=\left\{\begin{array}{cc}
\sum_{t \in T} \beta^{t} \frac{\tilde{\pi}_{t}}{\tilde{\pi}_{0}} \frac{\left(v_{t}(c ; D)\right)^{1-\rho}}{1-\rho} & \rho \geq 0, \rho \neq 1 \\
\sum_{t \in T} \beta^{t} \frac{\tilde{\pi}_{t}}{\tilde{\pi}_{0}} \ln \left(v_{t}(c ; D)\right) & \rho=1
\end{array}\right.
$$

\section{S2 implies $S 1$}

I show that $S 2$ implies that the social ordering function $\succsim$ satisfies risk balancing and intergenerational equity. The implication for strict monotonicity and proportionality are straightforward and are thus omitted.

Lemma 2. Assume S2. Then the social ordering function $\succsim$ satisfies risk balancing.

Proof. Let $D \in \mathcal{D}$. Let a pair $c, \bar{c} \in C(D)$ be such that for some $t \in T$, a pair $n, n^{\prime} \in N_{t}$ and a $\delta \in \mathbb{R}_{+}$the following conditions hold: (i) $c_{n}=\bar{c}_{n}-\frac{\delta}{\pi_{n}} \geq x_{n}$; (ii) 
$c_{n^{\prime}}=\bar{c}_{n^{\prime}}+\frac{\delta}{\pi_{n^{\prime}}} \leq x_{n^{\prime}} ;$ (iii) $c_{n^{\prime \prime}}=\bar{c}_{n^{\prime \prime}}$ for each $n^{\prime \prime} \neq n, n^{\prime}$. Let $\Delta \equiv \frac{\left(\frac{\bar{c}_{n}}{x_{n}}\right)^{\gamma}-\left(\frac{\bar{c}_{n^{\prime}}}{x_{n^{\prime}}}\right)^{\gamma}}{\frac{\bar{c}_{n}}{x_{n^{\prime}}}-\frac{c_{n^{\prime}}}{x_{n^{\prime}}}}$. Since $\gamma_{t}<1$,

$$
\begin{aligned}
& \left(\frac{c_{n}}{x_{n}}\right)^{\gamma}=\left(\frac{\bar{c}_{n}}{x_{n}}-\frac{\delta}{\pi_{n} x_{n}}\right)^{\gamma}>\left(\frac{\bar{c}_{n}}{x_{n}}\right)^{\gamma}-\frac{\delta}{\pi_{n} x_{n}} \Delta \text { and } \\
& \left(\frac{\bar{c}_{n^{\prime}}}{x_{n^{\prime}}}\right)^{\gamma}=\left(\frac{\bar{c}_{n^{\prime}}}{x_{n^{\prime}}}+\frac{\delta}{\pi_{n^{\prime}} x_{n^{\prime}}}\right)^{\gamma}>\left(\frac{\bar{c}_{n^{\prime}}}{x_{n^{\prime}}}\right)^{\gamma}+\frac{\delta}{\pi_{n^{\prime}} x_{n^{\prime}}} \Delta .
\end{aligned}
$$

Premultiply the first by $\pi_{n} x_{n}$ and the second by $\pi_{n^{\prime}} x_{n^{\prime}}$, adding up and simplifying, gives:

$$
\pi_{n} x_{n}\left(\frac{c_{n}}{x_{n}}\right)^{\gamma}+\pi_{n^{\prime}} x_{n^{\prime}}\left(\frac{c_{n^{\prime}}}{x_{n^{\prime}}}\right)^{\gamma}>\pi_{n} x_{n}\left(\frac{\bar{c}_{n}}{x_{n}}\right)^{\gamma}+\pi_{n^{\prime}} x_{n^{\prime}}\left(\frac{\bar{c}_{n^{\prime}}}{x_{n^{\prime}}}\right)^{\gamma} .
$$

By the additive structure of the representation, it follows that $V(c ; D)>V(\bar{c} ; D)$ and risk-balancing holds.

Lemma 3. Assume S2. Then the social ordering function $\succsim$ satisfies intergenerational equity.

Proof. Let $D \in \mathcal{D}$. Let a pair $c, \bar{c} \in C(D)$ be such that for some $t, t^{\prime} \in T$, with $t^{\prime}>t$, and $\delta \in \mathbb{R}_{+}$the following conditions hold: (i) $\frac{c_{n}}{x_{n}}=\frac{\bar{c}_{n}}{x_{n}}-\frac{\delta}{\beta^{t} x_{n}} \geq 1$ for each $n \in N_{t}$; (ii) $\frac{c_{n^{\prime}}}{x_{n^{\prime}}}=\frac{\bar{c}_{n^{\prime}}}{x_{n^{\prime}}}+\frac{\delta}{\beta^{t^{\prime} x_{n^{\prime}}}} \leq 1$ for each $n^{\prime} \in N_{t^{\prime}} ;$ (iii) $c_{n^{\prime \prime}}=\bar{c}_{n^{\prime \prime}}$ for each $n^{\prime \prime} \in N \backslash\left\{N_{t} \bigcup N_{t^{\prime}}\right\}$. Let:

$$
\begin{gathered}
y_{t} \equiv\left(\sum_{n \in N_{t}} \frac{\pi_{n} x_{n}}{\sum_{\bar{n} \in N_{t}} \pi_{\bar{n}} x_{\bar{n}}}\left(\frac{c_{n}}{x_{n}}\right)^{\gamma}\right)^{\frac{1}{\gamma}}, \bar{y}_{t} \equiv\left(\sum_{n \in N_{t}} \frac{\pi_{n} x_{n}}{\sum_{\bar{n} \in N_{t}} \pi_{\bar{n}} x_{\bar{n}}}\left(\frac{\bar{c}_{n}}{x_{n}}\right)^{\gamma}\right)^{\frac{1}{\gamma}}, \\
y_{t^{\prime}} \equiv\left(\sum_{n^{\prime} \in N_{t^{\prime}}} \frac{\pi_{n^{\prime}} x_{n^{\prime}}}{\sum_{\bar{n} \in N_{t^{\prime}}} \pi_{\bar{n}} x_{\bar{n}}}\left(\frac{c_{n^{\prime}}}{x_{n^{\prime}}}\right)^{\gamma}\right)^{\frac{1}{\gamma}}, \bar{y}_{t^{\prime}} \equiv\left(\sum_{n^{\prime} \in N_{t^{\prime}}} \frac{\pi_{n^{\prime}} x_{n^{\prime}}}{\sum_{\bar{n} \in N_{t^{\prime}}} \pi_{\bar{n}} x_{\bar{n}}}\left(\frac{\bar{c}_{n^{\prime}}}{x_{n^{\prime}}}\right)^{\gamma}\right)^{\frac{1}{\gamma}},
\end{gathered}
$$

and $\Delta \equiv \frac{1}{1-\rho} \frac{y_{t}^{1-\rho}-y_{t^{\prime}}^{1-\rho}}{y_{t}-y_{t^{\prime}}}$. For each $n \in N_{t}$ and each $n^{\prime} \in N_{t^{\prime}}(n)$, let $\Delta^{n, n^{\prime}} \equiv \frac{y_{t}-y_{t^{\prime}}}{\frac{\bar{c}_{n}}{x_{n}}-\frac{c_{n} n^{\prime}}{x_{n^{\prime}}}}$. Then, since $\rho \leq 1$ and $\gamma<1$ :

$$
\begin{aligned}
\frac{y_{t}^{1-\rho}}{1-\rho} & =\frac{1}{1-\rho}\left(\sum_{n \in N_{t}} \frac{\pi_{n} x_{n}}{\sum_{\bar{n} \in N_{t}} \pi_{\bar{n} x_{\bar{n}}}}\left(\frac{\bar{c}_{n}}{x_{n}}-\frac{\delta}{\beta^{t} x_{n}}\right)^{\gamma}\right)^{\frac{1-\rho}{\gamma}} \\
& \geq \frac{\bar{y}_{t}^{1-\rho}}{1-\rho}-\Delta \sum_{n \in N_{t}, n^{\prime} \in N_{t^{\prime}}(n)} \Delta^{n, n^{\prime}} \frac{\delta}{\beta^{t} x_{n}}
\end{aligned}
$$

and

$$
\begin{aligned}
\frac{y_{t^{\prime}}^{1-\rho}}{1-\rho} & =\frac{1}{1-\rho}\left(\sum_{n^{\prime} \in N_{t^{\prime}}} \frac{\pi_{n^{\prime}} x_{n^{\prime}}}{\sum_{\bar{n} \in N_{t^{\prime}}} \pi_{\bar{n} x_{\bar{n}}}}\left(\frac{\bar{c}_{n^{\prime}}}{x_{n^{\prime}}}+\frac{\delta}{\beta^{t} x_{n^{\prime}}}\right)^{\gamma}\right)^{\frac{1-\rho}{\gamma}} \\
& \geq \frac{\bar{y}_{t^{\prime}}^{1-\rho}}{1-\rho}+\Delta \sum_{n \in N_{t}, n^{\prime} \in N_{t^{\prime}}(n)} \Delta^{n, n^{\prime}} \frac{\delta}{\beta^{t^{\prime}} x_{n^{\prime}}} .
\end{aligned}
$$


Multiplying the first by $\beta^{t}\left(\sum_{n \in N_{t}} \pi_{n} x_{n}\right)$ and the second by $\beta^{t^{\prime}}\left(\sum_{n^{\prime} \in N_{t^{\prime}}} \pi_{n^{\prime}} x_{n^{\prime}}\right)$, rearranging (remember that $\pi_{n}=\sum_{n^{\prime} \in N_{t^{\prime}}(n)} \pi_{n^{\prime}}$ ), and summing up, gives:

$$
\beta^{t}\left(\sum_{n \in N_{t}} \pi_{n} x_{n}\right)\left[\frac{y_{t}^{1-\rho}}{1-\rho}-\frac{\bar{y}_{t}^{1-\rho}}{1-\rho}\right]+\beta^{t^{\prime}}\left(\sum_{n^{\prime} \in N_{t^{\prime}}} \pi_{n^{\prime}} x_{n^{\prime}}\right)\left[\frac{y_{t^{\prime}}^{1-\rho}}{1-\rho}-\frac{\bar{y}_{t^{\prime}}^{1-\rho}}{1-\rho}\right] \geq 0 .
$$

Substituting the variables and by the additive structure of the fair intergenerational utilitarian welfare function, it follows that $V(c ; D) \geq V(\bar{c} ; D)$ and intergenerational equity holds. The proof for the case where $t>t^{\prime}$ is similar and thus omitted.

\section{Appendix C. (Online): Accounting for the possibility of extinction}

In this online appendix, I present a more general version of the model, allowing for a positive probability of extinction. This extension also shows how to endogenize the pure time discount factor $\beta$. A decision tree is now denoted by:

$$
D^{+} \equiv\left\langle\left\langle\pi_{n}, F_{n}\right\rangle_{n \in N}, \underline{k}\right\rangle
$$

Each element of $D^{+}$is defined as follows. As before, $\underline{k} \in \mathbb{R}_{+}$denotes initial resources and, for each period $t \in T$ and node $n \in N_{t}, \pi_{n} \in(0,1]$ is the probability that node $n$ is reached at $t$. Technology at each $n \in N$ is now defined by $F_{n} \in \mathcal{F} \cup \emptyset$ : the case $F_{n}=\emptyset$ signifies that the human race is extinct in $n$; clearly, $F_{n}=\emptyset$ implies that $F_{n^{\prime}}=\emptyset$ for each $n^{\prime} \in N(n)$. Let $N^{n e} \subseteq N$ be the no-extinction subtree; it is such that $n \in N^{n e}$ only if $F_{n} \notin \emptyset$. Assume also that extinction is never sure: for each $n \in N^{n e}$, there exists $n^{\prime} \in N_{+1}(n)$ such that $n^{\prime} \in N^{n e}$. Let $\mathcal{D}^{+}$be this larger domain of decision trees $\left(\mathcal{D}\right.$ is obtained when $\left.N=N^{n e}\right)$.

A (risky) prospect for $\mathbf{D}^{+}$is denoted by $c \equiv\left(\left\{c_{n}\right\}_{n \in N^{n e}}\right) \in C\left(D^{+}\right) \equiv \mathbb{R}_{+}^{\left|N^{n e}\right|}$. For each $D^{+} \in \mathcal{D}^{+}$, let the set of feasible prospects for $\mathbf{D}^{+}$be $C^{F}\left(D^{+}\right) \subset C\left(D^{+}\right)$ such that $c \in C^{F}\left(D^{+}\right)$if for each $n \in N^{n e}$ : (i) $y_{n}=F_{n}\left(k_{n}\right)$; (ii) $c_{n}+s_{n} \leq y_{n}$; (iii) $k_{n^{\prime}}=s_{n}$ for each $n^{\prime} \in N_{+1}(n)$; iv) $k_{0}=\underline{k}$. The definition of the social ordering function is not affected by the introduction of the extinction probability and is not repeated for the sake of brevity.

C.1. Dealing with extinction probabilities: the fair prospect. How would an egalitarian planner distribute the resources, when facing a positive probability of extinction? The answer depends whether a generation should be compensated or not for having a lower probability of coming into existence. 
First note that maximality remains compelling for selecting the egalitarian reference: the prospect assigns consumptions only at nodes with living conditional generations; thus, the axiom only requires that it should not be possible to give more resources to these conditional generations.

The treatment of the generation in extinction nodes $\left(N \backslash N^{n e}\right)$ becomes instead important for adapting interim egalitarianism to this setting. Following Golosov et al. (2007), two alternatives can be formulated: (i) either the consumption of a generation in a non-existing node is exogenously set to a critical level or (ii) the comparison between generations is conditional on non-extinction. I follow the second alternative. The reason is that conditional generations are born after the technological/extinction shock is realized: since they do not face the extinction risk, one should only ensure that these are treated fairly when they come into existence.

As an example assume a unit of good is to be shared among two generations, the second of which comes to existence with probability $\frac{1}{3}$. Assume the egalitarian planner is risk-neutral and that the critical level is zero. Then, according to alternative (i), these generations are treated equally when the first is assigned .25 and the second .75; according to alternative (ii) instead, these generations are treated equally when each is assigned $\frac{1}{2}$. As discussed later, the willingness of the planner to save up more or less resources for later generations, reflecting the risk that future generations might not benefit from such effort, is introduced in the intergenerational transfer principle.

Let $\mu: \mathbb{R} \rightarrow \mathbb{R}$ be a continuous, strictly increasing, and concave function.

Conditional interim egalitarianism. For each $D^{+} \in \mathcal{D}^{+}, x \in \phi\left(D^{+}\right)$is such that for each $t \in[0, \bar{t}-1]$, each $n \in N_{t}^{n e}$, and each $t^{\prime}>t$ :

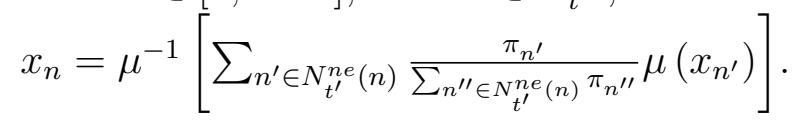

Define the fair rule $\phi^{*}$ as the one that satisfies maximality and conditional interim egalitarianism. This rule is well-behaved and selects a unique prospect that assigns strictly positive consumptions to all conditional generations at non-extinction nodes. The proof is similar to those of Proposition 1 and Corollary 1 and is omitted.

Proposition 2. The fair rule $\phi^{*}$ associates to each decision tree $D^{+} \in \mathcal{D}^{+}$a unique prospect $\phi^{*}\left(D^{+}\right)$such that $x_{n}^{*}>0$ for each $n \in N^{n e}$.

As for the model without extinction, the representation result is independent of the reference prospect, provided it is uniquely selected and assigns positive consumptions to all living conditional generations. 
C.2. Dealing with extinction probabilities: intergenerational equity. As for maximality, strict monotonicity remains compelling as extinction probabilities are given and just requires to value positively the assignment of larger prospects. Also risk-balancing remains appealing: the mean preserving transfer relative to the fair prospect should hold among histories where conditional generations are assigned a consumption, thus excluding the extinction nodes. Finally, also the axiom of proportionality remains compelling.

As anticipated, the introduction of extinction probabilities requires to opportunely adapt the axiom of intergenerational equity. In fact, the Pigou-Dalton type of progressive transfers between generations, uniform across histories, might not be considered anymore welfare improving when the recipient generation has a very low probability of benefiting from the transfer.

Compared to the corresponding axiom of Section 3, I suggest using the information about the probability of extinction to infer how to discount the transfer across generations. Assume one generation is richer than another: at each history the first is assigned more than the fair prospect and the second is assigned less. Consider a progressive transfer, uniform across histories and weighted by the likelihood of existence of the generation. Then, transferring such (weighted) amounts from the richer to the poorer generation without changing the richer-poorer relation, leads to a larger social welfare. Formally, the probability of a generation being alive substitutes the pure time discount factor, i.e. for each $t \in T$ the transfer $\delta \in \mathbb{R}_{+}$is weighted by $\sum_{\bar{n} \in N_{t}^{n e} \pi_{\bar{n}}}$ instead of $\beta^{t}$. The fair prospect is here uniquely selected by the function $\psi: \mathcal{D}^{+} \rightarrow C^{F}\left(\mathcal{D}^{+}\right) \bigcap \mathbb{R}_{++}^{N^{n e}}$ (redefined on the larger domain $\mathcal{D}^{+}$).

Conditional intergenerational equity. For each $D^{+} \in \mathcal{D}^{+}$, each $x=\psi\left(D^{+}\right)$, each pair $c, \bar{c} \in C\left(D^{+}\right)$, each pair $t, t^{\prime} \in T$, and each $\delta \in \mathbb{R}_{+}$, if

then

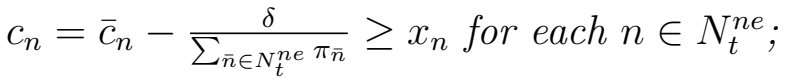

$$
\begin{aligned}
& c_{n^{\prime}}=\bar{c}_{n^{\prime}}+\frac{\delta}{\sum_{\bar{n} \in N_{t^{\prime}}^{n e} \pi_{\bar{n}}}} \leq x_{n^{\prime}} \text { for each } n^{\prime} \in N_{t^{\prime}} \text {; } \\
& c_{n^{\prime \prime}}=\bar{c}_{n^{\prime \prime}} \text { for each } n^{\prime \prime} \in N^{n e} \backslash\left\{N_{t}^{n e} \bigcup N_{t^{\prime}}^{n e}\right\} \text {, } \\
& c \succsim_{D^{+}} \bar{c} \text {. }
\end{aligned}
$$

C.3. The characterization result. Let $D^{+} \in \mathcal{D}^{+}$and $x=\psi\left(D^{+}\right)$. For each generation $t \in T$, the risk-and-extinction-adjusted discount factor is:

$$
\hat{\beta}_{t} \equiv\left(\sum_{n \in N_{t}^{n e}} \pi_{n}\right)\left(\frac{\sum_{n \in N_{t}^{n e}} \pi_{n} x_{n}}{x_{0}}\right)
$$


and let generation t's welfare be measured by the function $v_{t}: \mathbb{R}_{+}^{\left|N_{t}^{n e}\right|} \rightarrow \mathbb{R}$ with the following functional form:

$$
v_{t}\left(c ; D^{+}\right) \equiv\left\{\begin{array}{cc}
\left(\sum_{\left.n \in N_{t}^{n e} \frac{\pi_{n} x_{n}}{\sum_{\bar{n} \in N_{t}^{n e} \pi_{\bar{n}} x_{\bar{n}}}}\left(\frac{c_{n}}{x_{n}}\right)^{\gamma}\right)^{\frac{1}{\gamma}}}\right. & \gamma<1, \gamma \neq 0 \\
\prod_{n \in N_{t}^{n e}}\left(\frac{c_{n}}{x_{n}}\right)^{\sum_{\bar{n} \in N_{n}^{n e} \pi_{\bar{n}} x_{\bar{n}}}} & \gamma=0
\end{array}\right.
$$

Extended fair intergenerational utilitarianism: For each $D^{+} \in \mathcal{D}^{+}$,

$$
V\left(c ; D^{+}\right) \equiv\left\{\begin{array}{cc}
\sum_{t \in T} \hat{\beta}_{t} \frac{\left(v_{t}\left(c ; D^{+}\right)\right)^{1-\rho}}{1-\rho} & \rho \geq 0, \rho \neq 1 \\
\sum_{t \in T} \hat{\beta}_{t} \ln \left(v_{t}\left(c ; D^{+}\right)\right) & \rho=1
\end{array}\right.
$$

where, for each $t \in T, \hat{\beta}_{t}$ is defined in $(\mathrm{C} .1)$ and $v_{t}\left(c ; D^{+}\right)$in $(\mathrm{C} .2)$.

Theorem 2. On the domain $\mathcal{D}^{+}$, the following statements are equivalent:

$S 1$ : $\quad$ the social ordering function $\succsim$ satisfies strict monotonicity, conditional intergenerational equity, risk-balancing, and proportionality;

$S 2$ : $\quad$ for each $D^{+} \in \mathcal{D}^{+}$, the social ordering $\succsim_{D^{+}}$can be represented by the extended fair intergenerational utilitarian criterion (C.3).

Proof. The proof is similar to the one of Theorem 1 and is omitted.

\section{REFERENCES}

Anthoff, D., R. S. Tol, And G. W. Yohe (2009): "Risk aversion, time preference, and the social cost of carbon," Environmental Research Letters, 4, 024002.

Arrow, K. J. And A. C. Fisher (1974): "Environmental preservation, uncertainty, and irreversibility," The Quarterly Journal of Economics, 88, 312-319.

Asheim, G. B. And K. A. BrekKe (2002): "Sustainability when capital management has stochastic consequences," Social Choice and Welfare, 19, 921-940.

Asheim, G. B. AND S. Zuber (2013): "Probability adjusted rank-discounted utilitarianism," CESifo Working Paper Series No. 4728., Available at SSRN: http://ssrn.com/abstract $=2428638$.

Atkinson, A. B. (1970): "On the measurement of inequality," Journal of economic theory, $2,244-263$.

Blackorby, C. And D. Donaldson (1982): "Ratio-scale and translation-scale full interpersonal comparability without domain restrictions: Admissible social-evaluation functions," International Economic Review, 249-268.

Bossert, W. And J. A. Weymark (2004): "Utility in social choice," in Handbook of Utility Theory, ed. by S. Barberà, P. Hammond, and C. Seidl, Springer, 1099-1177. 
Broome, J. (1984): "Uncertainty and fairness," Economic journal, 94, 624-632.

- (1991): Weighing goods: Equality, uncertainty and time, Basil Blackwell Oxford.

Dalton, H. (1920): "The Measurement of the Inequality of Incomes," The Economic Journal, 30, 348-361.

DAsGupta, P. (2008): "Discounting climate change," Journal of risk and uncertainty, 37, 141-169.

De Finetti, B. (1931): "Sul Concetto di Media," Giornale dell'Istituto Italiano degli Attuari, 2, 369-396.

Diamond, P. A. (1967): "Cardinal welfare, individualistic ethics, and interpersonal comparison of utility: Comment," The Journal of Political Economy, 75, 765-766.

Fleurbaey, M. (2010): "Assessing Risky Social Situations," Journal of Political Economy, $118,649-680$.

Fleurbaey, M. And F. Maniquet (2011): A Theory of Fairness and Social Welfare, Cambridge University Press.

Fleurbaey, M. And S. Zuber (2014a): "Discounting, beyond utilitarianism," Princeton University William S. Dietrich II Economic Theory Center Research Paper No. 060., Available at SSRN: http://ssrn.com/abstract=2391853.

- (2014b): "Discounting, Risk and Inequality: A General Approach," CES Working Paper, No 2014.15.

Gerlagh, R. And M. Liski (2013): "Carbon prices for the next thousand years," mimeo.

Golosov, M., L. E. Jones, And M. Tertilt (2007): "Efficiency with endogenous population growth," Econometrica, 75, 1039-1071.

Gorman, W. (1968): "Conditions for additive separability," Econometrica, 36, 605-609.

Grant, S., A. KajiI, B. Polak, And Z. SAfra (2012): "Equally-distributed equivalent utility, ex post egalitarianism and utilitarianism," Journal of Economic Theory, 147, 15451571.

Hammitt, J. K., R. J. Lempert, And M. E. Schlesinger (1992): "A sequential-decision strategy for abating climate change," Nature, 357, 315-318.

HANEmann, W. M. (1989): "Welfare evaluations in contingent valuation experiments with discrete response data: reply," American Journal of Agricultural Economics, 71, 10571061.

Hardy, G. H., J. E. Littlewood, And G. Polya (1934): Inequalities, Cambridge University Press.

HARsANYi, J. C. (1953): "Cardinal utility in welfare economics and in the theory of risktaking," The Journal of Political Economy, 61, 434-435.

(1955): "Cardinal Welfare, Individual- istic Ethics, and Interpersonal Comparisons of Utility," Journal of Political Economy, 63, 309-321. 
Harstad, B. (2013): "Investment Policy for Time-Inconsistent Discounters," CESifo Working Paper Series No. 4546.

HenRY, C. (1974): "Investment decisions under uncertainty: the" irreversibility effect"," The American Economic Review, 64, 1006-1012.

Jackson, M. O. And L. YARIV (2014): "Collective Dynamic Choice: The Necessity of Time Inconsistency," American Economic Journal: Microeconomics, forthcoming.

Kahneman, D. And A. Tversky (1979): "Prospect theory: An analysis of decision under risk," Econometrica, 47, 263-291.

KARP, L. (2005): "Global warming and hyperbolic discounting," Journal of Public Economics, 89, 261-282.

Koopmans, T. C. (1960): "Stationary ordinal utility and impatience," Econometrica, 28, 287-309.

Kreps, D. M. And E. L. Porteus (1978): "Temporal resolution of uncertainty and dynamic choice theory," Econometrica, 46, 185-200.

Kòszegi, B. And M. RABin (2006): "A model of reference-dependent preferences," The Quarterly Journal of Economics, 121, 1133-1165.

LAibson, D. (1997): "Golden eggs and hyperbolic discounting," The Quarterly Journal of Economics, 112, 443-478.

Millner, A. (2013): "On welfare frameworks and catastrophic climate risks," Journal of Environmental Economics and Management, 65, 310-325.

Nordhaus, W. D. (2011): "The economics of tail events with an application to climate change," Review of Environmental Economics and Policy, 5, 240-257.

Piacquadio, P. G. (2014): "Intergenerational Egalitarianism," Journal of Economic Theory, 153, 117-127.

Pigou, A. C. (1912): Wealth and Welfare, London: Macmillan.

PINDYCK, R. S. (2000): "Irreversibilities and the timing of environmental policy," Resource and energy economics, 22, 233-259.

Rothschild, M. And J. E. Stiglitz (1970): "Increasing risk: I. A definition," Journal of Economic Theory, 2, 225-243.

STERn, N. (2013): "The structure of economic modeling of the potential impacts of climate change: grafting gross underestimation of risk onto already narrow science models," Journal of Economic Literature, 51, 838-859.

Sugden, R. (2003): "Reference-dependent subjective expected utility," Journal of economic theory, 111, 172-191.

Thomson, W. (2011): "Chapter Twenty-One - Fair Allocation Rules," in Handbook of Social Choice and Welfare, ed. by A. S. Kenneth J. Arrow and K. Suzumura, Elsevier, vol. 2 of Handbook of Social Choice and Welfare, 393-506. 
Traeger, C. P. (2012): "Once Upon a Time Preference - How Rationality and Risk Aversion Change the Rationale for Discounting," CESifo Working Paper Series No. 3793. Weitzman, M. L. (2001): "Gamma discounting," American Economic Review, 91, 260-271. - (2009): "On modeling and interpreting the economics of catastrophic climate change," The Review of Economics and Statistics, 91, 1-19.

(2011): "Fat-tailed uncertainty in the economics of catastrophic climate change," Review of Environmental Economics and Policy, 5, 275-292.

ZuBER, S. (2011): "The aggregation of preferences: can we ignore the past?" Theory and Decision, 70, 367-384.

E-mail address: p.g.piacquadio@econ.uio.no

University of Oslo, Moltke Moes vei 31, 0851, Oslo, Norway 